\title{
Factores climáticos que influyen en la diversidad de insectos en Spartium junceum L. (Fabales: Fabaceae)
}

\section{Climatic factors that influence the diversity of insects in Spartium junceum L. (Fabales: Fabaceae)}

\author{
Hernán Baltazar C." \\ ${ }^{(1)}$ Facultad de Ciencias Forestales y del Ambiente, Universidad Nacional del Centro del Perú \\ Email: hbaltaza@hotmail.com
}

\section{Resumen}

El Spartium junceum L., es una leguminosa distribuida en el valle del Mantaro, y está en peligro de extinción por severos daños ocasionado por afidos. Se investigó la influencia de factores climáticos en la diversidad de insectos en la "retama". Se atrapó, cuantificó e identificó durante el año 20l0, con muestreo intencionado, las provincias de Chupaca (Tres de Diciembre), Concepción (Orcotuna), Jauja (El Mantaro) y Huancayo (Cochas Grande). Los datos del clima fueron proporcionados por el Servicio Nacional de Meteorología e Hidrología (SENAMHI). Para el valle del Mantaro el número promedio de insectos fue de 151। 1 ; y cuyas medias fueron estadísticamente similares en las cuatro provincias. El índice de Simpson fue 0.70; índice de Shannon fue 1.94; el índice de Margalef fue 7.13 y el índice de Berger - Parker fue 0.46. En Concepción hubo mayor dominancia para las familias observadas. Con el índice Simpson, la población de insectos en Chupaca y Jauja tuvo una mayor dominancia y menor diversidad. Huancayo y Concepción tienen menor dominancia y mayor diversidad. Según el Índice de Margalef, la riqueza específica es mayor en Jauja y Chupaca. Con el índice de Shannon la diversidad es baja y heterogénea. Los factores climáticos influyen en la disminución de la diversidad de insectos en la "retama" cuando la precipitación aumentó; y cuando la humedad relativa y horas sol disminuyo la diversidad también aumentó. Se identificaron 10 órdenes, 32 familias, 35 géneros y 16 especies de insectos. Para el índice de Margalef, a mayor humedad relativa fue menor diversidad y heterogeneidad, y mayor dominancia. La "retama" hospeda una diversidad de órdenes, familias y especies de insectos valiosa para su recuperación.

Palabras claves: diversidad insectos, factor clima, Spartium junceum L.

\begin{abstract}
The Spartium junceum L., is a legume distributed in the Mantaro valley, and is in danger of extinction due to severe damage caused by aphids. The influence of climatic factors on the diversity of insects in the "broom" was investigated. The provinces of Chupaca (Tres de Diciembre), Concepción (Orcotuna), Jauja (El Mantaro) and Huancayo (Cochas Grande) were trapped, quantified and identified during the year 2010, with intentional sampling. The climate data was provided by the National Meteorology and Hydrology Service (SENAMHI). For the Mantaro valley, the average number of insects was I5IIII; and whose means were statistically similar in the four provinces. The Simpson index was 0.70; Shannon's index was 1.94; the Margalef index was 7.13 and the Berger - Parker index was 0.46. In Concepción there was greater dominance for the families observed. With the Simpson index, the insect population in Chupaca and Jauja had a greater dominance and less diversity. Huancayo and Concepción have lower dominance and greater diversity. According to the Margalef Index, the specific wealth is greater in Jauja and Chupaca. With the Shannon index, diversity is low and heterogeneous. Climatic factors influence the decrease in the diversity of insects in the "broom" when rainfall increased; and when relative humidity and sun hours decreased diversity also increased. Ten orders, 32 families, 35 genera and 16 insect species were identified. For the Margalef index, the higher the relative humidity, the lower the diversity and heterogeneity, and the greater the dominance. The "retama" hosts a variety of orders, families and species of insects valuable for their recovery.
\end{abstract}

Keywords: diversity insects, weather factor, Spartium junceum $L$. 


\section{Introducción}

Los insectos son considerados buenos indicadores de niveles de impacto ambiental, debido a su gran diversidad de especies y hábitat, también a su importancia a los procesos biológicos de los ecosistemas naturales. (Thomanzini y Thomanzini, 2002).

La interacción planta-herbívoro es de particular interés agrícola, como también el flujo potencial de carbón y nutrientes que afecta al ecosistemas (Frost and Hunter, 2004) y por lo tanto puede afectar la emisión del CO2 atmosférico. Estudios de laboratorio concluyen que la abundancia de insectos con el aumento de la temperatura (Cannon, 1998; Bale et al., 2002), y además la temperatura es la variable climática del cambio climático global, que directamente afecta a los insectos herbívoros (Bale et al., 2002). Aunque los insectos responden a la variación del cambio climático global, los efectos del calentamiento global sobre la interacción insecto - planta han sido generalmente observadas por la presión de los herbívoros sobre las plantas. (Cannon, 1998; Coley, 1998; Wilf y Labandeira, 1999; Bale et al., 2002).

Los (Hemíptera: Aphididae) son insectos herbívoros, en donde el crecimiento y abundancia puede incrementar o disminuir, o no ser afectado por el calentamiento global. La densidad de los insectos puede regular la densidad de los pulgones sobre otros insectos succionadores en dos modelos estudiados concluyendo que gradiente elevada de temperatura incrementa la densidad del insecto en la abundancia. Sin embargo, el incremento de la temperatura, puede también disminuir el crecimiento de algunas especies de pulgones, dependiendo de su requerimiento termal y especifidad del hospedante (Bale et al., 2002).

La "retama" (S. junceum L), es una especie leguminosa, de interés ecológico, ornamental, medicinal y energético; y está adaptada al clima de la región quechua, y es una especie apreciada por los habitantes, por el rol ecológico que cumple, además de incorporar nitrógeno al suelo, con un valor ornamental, energético y medicinal que beneficia al poblador (Brack, y Mendiola, 2004). Cumple una función ambiental en el ecosistema, y cuya utilización tiene importancia social y económica en el valle del Mantaro. Actualmente, hay 836 hectáreas de arbustos de "retama", plantado a una densidad media de 205 plantas por hectárea, y tiene buena capacidad de brotamiento; y dichas plantas está infestada por pulgones. (SENASA, 2006). Esta especie, alberga a insectos benéficos y perjudiciales. (Catalán et al., 2006), está atacado por pulgones siendo las especies Aphis craccivora, Aphis cystisorum (plaga clave), y el Aphis fabae, y ocasionando pérdida del valor ornamental y ecológico, (Vilca y Vergara, 20 I I) y (Senasa, 2008).

El valle del Mantaro, comprende las provincias de Jauja, Concepción, Huancayo y Chupaca respectivamente, tiene zonas de vida con un clima variado de templado a frio durante el año. Se localiza desde 3100 hasta 4680 msnm (Holdridge, 1987). Está afectada por el cam- bio climático, y en los últimos 90 años, la tendencia a largo plazo se observó que la temperatura se incrementó

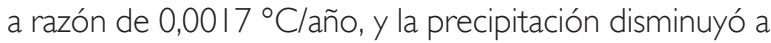
razón de 0,0013 mm/año, observando que hubo mayor calor y sequia (Arroyo, J. 20II). Se ha observado un incremento de la temperatura media anual de 2,44oC, y disminución de la precipitación media anual de 23,92 mm de 1998 al 2007 (Senamhi, 2008). Además de junio 1983 hasta diciembre de 1992, el incremento de CO2, esta sobre 0,9 ppm CO2/año, y ha producido un ligero incremento de la temperatura. (Tazza, C. y Suárez, L. 2004).

Este comportamiento climático, afectó a la adaptación y crecimiento, favoreciendo la invasión por insectos y como consecuencia disminuyo la población de algunos insectos controladores biológicos nativos, y situando en peligro de extinción. (SENASA, 2006); (Baltazar, H. 2007). Además, manifiesta síntomas de marchitez generalizada, perdida vigor, recubrimiento por fumagina y finalmente muerte masiva, como consecuencia del daño ocasionado por los pulgones.

La "retama", crece junto a otras especies vegetales leñosas y arbustivas, y es refugio de una diversidad variada de insectos que aún no ha sido estudiada.

El S. junceum L., crece en el valle del Mantaro, y alberga a varias órdenes, familias y especies de insectos, siendo benéficos y/o perjudiciales, y esta atacado por una plaga muy severa de pulgones que ha situado en peligro de desaparición.

Aguilar (2009), ha reportado la presencia de insectos perjudiciales y benéficos, situando en riesgo su sanidad por el ataque de pulgones, e implicando la disminución importante rol ecológico, ornamental y ambiental que tiene la "retama".

La variación de los factores climáticos, en la última década ha afectado la dinámica poblacional de insectos en la "retama", alterando su ciclo de vida, tasa de reproducción y hábitos de alimentación, favoreciendo el aumento de fitófagos perjudiciales y disminución de insectos benéficos.

La investigación tuvo como objetivo, evaluar la influencia de los factores climáticos en la diversidad de insectos en $S$. junceum $L$, describiendo la composición y estructura de la fauna entomológica en S. junceum L.; y explicar los factores climáticos que influyen en la población de insectos en S. junceum L.; e identificar a géneros y especies de insectos observado en S. junceum L., para establecer una base científica, que en el futuro desarrollar y aplicar un plan de manejo integrado de plagas y enfermedades a fin de recuperar a dicha especie.

\section{Materiales y métodos}

La investigación se realizó en el ámbito del valle del Mantaro, en las provincias de Huancayo, Chupaca, Concepción y Jauja, realizando captura de insectos en plantas de $S$. junceum $L$. de enero a diciembre del año 2010. 
Se aplicó el método deductivo - inductivo, mediante el análisis - síntesis, para evaluar la influencia de los factores climáticos en la diversidad de insectos en plantas "retama" en el valle del Mantaro. (Hernández et al., 2006).

La investigación realizada fue no experimental y no probabilística. El número de insectos por orden y familia, se evaluó en tendencia longitudinal del uno de enero a 3 I diciembre del 2010.

Se describió la composición y estructura de la población de insectos; y se explicó la influencia de los factores climáticos de precipitación, humedad relativa y horas sol en la diversidad de insectos en la "retama".

Los indicadores de las variables del clima y diversidad, fue descriptiva y cuantitativa. Se evaluó la correlación del número de insectos observado y los factores climáticos. Se explicó la influencia de los factores climáticos sobre la diversidad aplicando la regresión lineal simple y múltiple. El muestreo aplicado fue intencionado (Hernández et al., 2006), eligiendo plantas de S. junceum L. representativa de cada provincia.

Se identificaron a órdenes, familias, géneros y especies de insectos en la "retama".'La población, estuvo constituida por plantas de "retama", cuyo estado fenológico fue con o sin floración, y por insectos imago observado al momento de la evaluación, situado en cada una de las provincias del valle del Mantaro.

El muestreo fue intencionado, determinando 20 plantas, asumiendo el 10 por ciento, del número promedio de planta existente por hectárea en el valle del Mantaro. (Senasa, 2006).

Se diseñaron e instalaron transectos, siendo la forma "W" en un área de 50 m2, para no cuantificar a los insectos que fueron observados en una observación anterior.

Las plantas de S. junceum L. estuvo ubicado en terrenos con pendiente entre 25 y $40 \%$. En cada provincia el área de las plantas muestreada se delimitó con una cinta.

La ubicación de los lugares de muestreo en cada provincia fue en la provincia Chupaca, distrito Tres de Diciembre, coordenadas UTM 472877.61 E y $8658881.21 \mathrm{~N}$, altitud $3196 \mathrm{msnm}$. En la provincia Concepción, distrito Orcotuna, coordenadas UTM 4658 I3.54 E y 867 I54.65 N, altitud 33 I I msnm. En la provincia Jauja, distrito El Mantaro, coordenadas UTM 457|37.96 E y 8691951.72 N, altitud 3321 msnm y en la provincia Huancayo, distrito El Tambo, Anexo Cochas Grande, coordenadas UTM 478905.88 E y 8673320.08 $\mathrm{N}$, altitud $3590 \mathrm{msnm}$.

Se instalaron los métodos de captura de insectos, mediante la lona, rastreo aéreo, trampas pitfall o de caída y trampas Moericke o pegantes aplicada por Briceño et al., (2005); Gaviño de la Torre et al., (2004); Santisteban y Aldana, (2005).

Los insectos fueron atrapados, siguiendo la recomendación de Ganho \& Marinoni (2003), lannacone, Alayo \& Sánchez (2000) y Sánchez (2003). Registrando cada 15 días en las fichas de campo.

Las variables de precipitación ( $\mathrm{mm}$ ); humedad relativa (\%) y horas sol (HS), fue registrada del uno de enero hasta el 3I de diciembre del año 2010 por Servicio Nacional de Meteorología e Hidrología, en las estaciones meteorológicas localizado en Jauja, Ingenio, Huayao, Santa Ana y Viques.

Se elaboró una base de datos con el software excel y SPSS 15.0; se obtuvo la estadística descriptiva; y luego se estimaron los índices de diversidad de la población de insectos utilizando el software Past. (Øyvind Hammer, 2012).

Para describir la composición y estructura de la fluctuación poblacional, se organizó la información del número de insectos por órdenes y familias observadas en cada provincia; luego se aplicó la prueba de normalidad de Kolmogorov - Smirnov $(\alpha=0.05)$; y finalmente se graficó abundancia mediante curva lognormal aplicado por Magurran, (1988) y Krebs (1978 y 1989).

Aplicando el software $R$, se estimó la eficiencia muestral, para el número de insectos registrado en las cuatro provincias muestreada. Además se obtuvo la curvas de rarefacción, aplicando y ajustando la ecuación asintótica aplicado por Clench, (1979). Siendo validado con el R2 y el criterio de información de Akaike (AIC). Se aplicó la prueba " $t$ " de contrastación de hipótesis a nivel $\alpha=0.05$ Hutcheson (1970), para comparar la diversidad y abundancia hallado con el índice de Shannon Wiener, de la población de insectos observado en cada provincia.

$$
t=\begin{array}{c|}
H_{1}^{\prime}-H_{2}^{\prime} \\
\hline S_{H^{\prime} 1}-S_{H^{\prime} 2}
\end{array}
$$

Donde $\mathrm{Hi}$ = valor del índice de diversidad del sitio y $\mathrm{SHi}$ = representa la varianza de diversidad del sitio

Para estimar la diversidad de insectos, se aplicaron los índices y parámetros de la abundancia $(\mathrm{N})$; riqueza, medida como el número de especies (S); índice de Margalef (DM), índice de diversidad de Shannon - Wiener $\left(H^{\prime}\right)$; índice de dominancia de Berger-Parker (d); el índice de Simpson $(\lambda)$, referido por Magurran (1988) y Moreno (200I).

Para explicar la influencia de los factores climáticos en la diversidad de insectos de la "retama", se aplicó regresión lineal y múltiple, relacionando las variables predictivas (precipitación, humedad relativa y horas sol) y variable respuesta (número de insectos); calculando los coeficientes la regresión. Además, se obtuvo los coeficientes de correlación (R) y determinación (R2), el análisis de varianza de la regresión. Finalmente se contrastó las hipótesis con la prueba F, a un nivel de significación 0,05. Se estimó aplicando regresión múltiple (Kuehl, 200 I; y Fresse, 1978) para tres variables independiente, expresada en el siguiente modelo:

$Y=B O+\beta|\times|+\beta 2 \times 2+\beta 3 \times 3+e$ 
donde:

$Y d=$ Número de insectos $\left(N^{\circ}\right)$

$X \mid=$ Precipitación media mensual $(\mathrm{mm})$

$\mathrm{X} 2$ = Humedad relativa media mensual (\%)

X3 = Horas sol media mensual (hr)

$\mathrm{e}=$ Error o residuo

3.6.I. Ecuación de regresión múltiple estimada.

Estuvo determinado por la ecuación:

$$
\hat{Y}=b o+b|X|+b 2 X 2+b 3 X 3
$$

donde:

bo = intercepto; bl, b2, y b3 = coeficientes de regresión parcial; $\quad X 1, \times 2$, y $\times 3$, = variables independientes

Las variables estudiadas fueron independiente el factor clima: precipitación ( $\mathrm{mm}$ ), humedad relativa (\%) y horas sol (hs); y dependiente la diversidad: riqueza, abundancia, dominancia, equidad y heterogeneidad

Población de insectos: número de individuos de insectos, composición y estructura; número de órdenes y familias. Luego de atrapar e inmovilizar a los insectos, se seleccionaron a los individuos adultos. Se preparó libre de residuos, y se ubicaron en tubos de ensayo que contenían alcohol al 70\%, y que fue taponado con goma. Se codificaron y etiquetaron, y se enviaron cinco individuos por género y/o especie, al Servicio Nacional de Sanidad Agraria, para confirmar la identificación de las órdenes, familias, géneros y especies colectada. Se tomó en cuenta la claves de identificación de Borror et al., (1995).

\section{Resultados y discusión}

Tabla I.

Composición y estructura de insectos en S. junceum L. en valle del Mantaro

\begin{tabular}{|c|c|c|c|c|c|}
\hline \multirow{2}{*}{ Ordenes } & \multicolumn{4}{|c|}{ Provincia } & \multirow{2}{*}{$\%$ total } \\
\hline & Chupaca & Huancayo & Concepción & Jauja & \\
\hline Odonata & 67 & 56 & 103 & 58 & 0,19 \\
\hline Blatodea & 62 & 42 & 29 & 44 & 0,12 \\
\hline Ortóptera & 168 & 451 & 314 & 412 & 0,89 \\
\hline Dermáptera & 55 & 52 & 23 & 25 & 0,10 \\
\hline Psocóptera & 554 & 102 & 45 & 3 & 0,47 \\
\hline Thysanóptera & 5303 & 13504 & 7072 & 20463 & 30,67 \\
\hline Hemíptera & 11262 & 14195 & 27365 & 20160 & 48,30 \\
\hline Neuróptera & 35 & 26 & 12 & 5 & 0,05 \\
\hline Coleóptera & 1378 & 1603 & 1320 & 1651 & 3,94 \\
\hline Diptera & 3896 & 4409 & 3057 & 2790 & 9,37 \\
\hline Lepidóptera & 149 & 110 & 120 & 54 & 0,29 \\
\hline Himenóptera & 1915 & 1961 & 2063 & 2568 & 5,63 \\
\hline Total & 24844 & 36511 & 41523 & 48233 & 100,00 \\
\hline
\end{tabular}

El análisis de la Tabla I, la composición y estructura poblacional estuvo distribuida en 12 ordenes, destacando un $48.30 \%$ los Hemípteros, luego Thysanóptera con 30,67\%; y siguiendo el orden de importancia los Dípteros con 9.37\%; los Himenópteros con 5.63; los coleópteros con 3.94\%; siendo las ordenes Blatodea con $0.12 \%$ y Dermáptera con 0,10\% de menor presencia poblacional. (Tabla I y Figura 2).

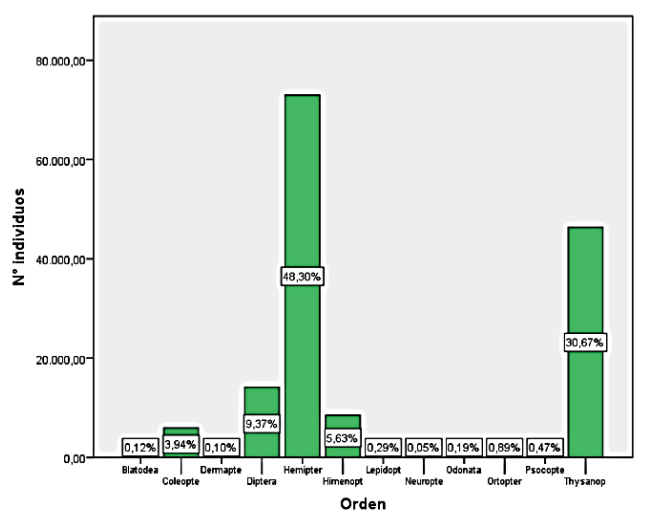

Figura 2. Distribución de la población de insectos en S. junceum $L$. en porcentaje

Tabla 2

Población de insectos por provincia muestreada en S. junceum L.

\begin{tabular}{ccccc}
\hline Mes & Chupaca & Huancayo & Concepción & Jauja \\
\hline Enero & 3692 & 7083 & 4801 & 3298 \\
Febrero & 1378 & 5069 & 4454 & 3109 \\
Marzo & 2884 & 2527 & 3162 & 3771 \\
Abril & 1208 & 1522 & 1000 & 5864 \\
Mayo & 1671 & 1190 & 2810 & 1753 \\
Junio & 998 & 825 & 3867 & 1472 \\
Julio & 1377 & 1493 & 1979 & 2496 \\
Agosto & 1155 & 1332 & 1128 & 1423 \\
Setiembre & 2992 & 3396 & 4674 & 4869 \\
Octubre & 1306 & 4066 & 3967 & 4887 \\
Noviembre & 2799 & 3655 & 4372 & 6990 \\
Diciembre & 3384 & 4353 & 5309 & 8300 \\
\hline Total & 24844 & 36511 & 41523 & 48233 \\
\hline 0 & 2070,33 & 30442,58 & 3460,25 & 4019,33 \\
\hline CV & 47,93 & 62,54 & 41,90 & 55,19 \\
\hline$\%$ & 16,44 & 24,16 & 24,78 & 31,92 \\
\hline
\end{tabular}

En la Tabla 2, se observa las distribución de insectos en S. junceum L. durante el año 20 I0; corresponde a la provincia de Chupaca 16,44\%, Huancayo 24,16\%, Concepción 24,78\% y Jauja 31,92\% del total de la población evaluada, siendo población relativamente mayor en Jauja y menor en Chupaca.

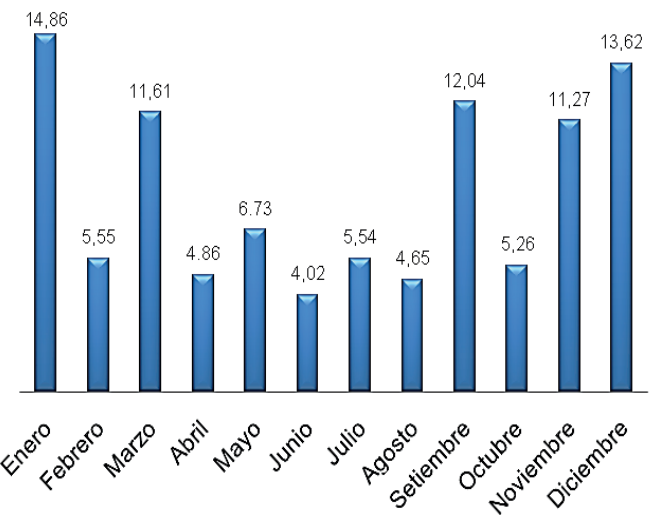

Figura 3. Distribución población insectos en Huancayo (\%) 
En la provincia de Huancayo, la población de insectos observada (Figura 3) en la "retama", tuvo relación con valores del clima registrado, manifestando que cuando el clima disminuyó, como la precipitación, temperatura y humedad relativa (Figura 13 y 14), la población de insectos también disminuyo. En los meses de enero, febrero setiembre, octubre, noviembre y diciembre variaron de $9.30 \%$ hasta $19.40 \%$; mientras que en periodo seco en los meses de abril a agosto varió de 2.26\% a $4.17 \%$ el número de individuos fue menor con relación a la provincia de Chupaca.

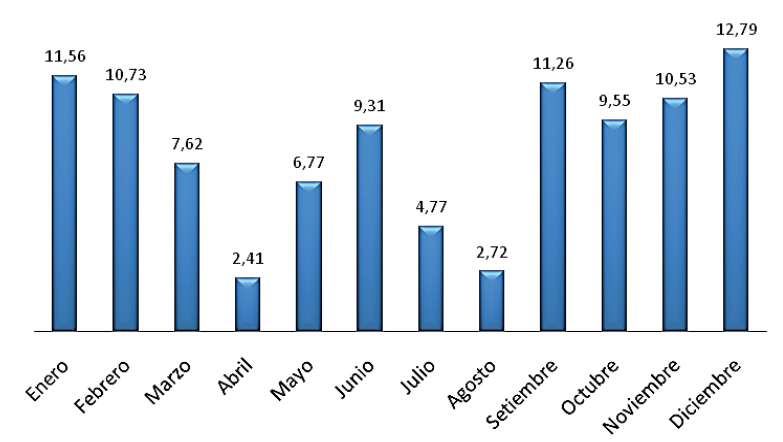

Figura 4. Distribución población insectos en Concepción (\%)

En la Figura 4, de los insectos observado en S. junceum L., la provincia de Concepción, tuvo el número de individuos mayor, y fue similar en los meses de enero, con 1 I.56\%; febrero con 10.73\%; junio con $9.31 \%$; setiembre con 11.26\%; octubre con 9.55\%; noviembre con $10.53 \%$ y diciembre con $12.79 \%$. Mientras que el número de individuos disminuyó en los meses de abril con $2.41 \%$; mayo con $6.77 \%$; julio con $4.77 \%$ y agosto con $2.72 \%$. Siendo de una característica similar a la observada en la provincia de Huancayo.

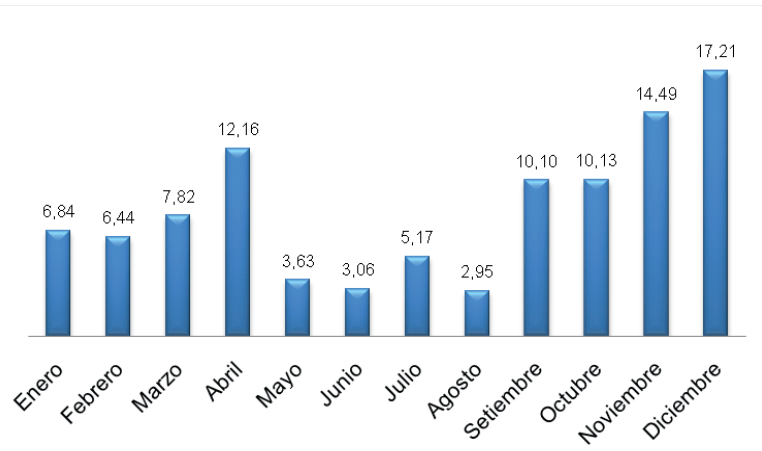

Figura 5. Distribución población insectos en Jauja (\%)

Analizando la Figura 5, la presencia de insectos en S. junceum L. en Jauja disminuyó considerablemente en los meses de enero con 6,84\%; febrero con 6,44\% y marzo con 7,62; siendo contrario para el mismo periodo evaluado en Chupaca, Concepción y Huancayo. Además la población de insectos por el número de individuos fue similar en el mes de mayo con 3,63\%; junio con 3,06\%; julio con $5,17 \%$ y agosto con 2,95\%; y tuvo un incre- mento considerable en el mes de setiembre con 10,10\%; octubre con 10,13\%; noviembre con 14,49\% y diciembre con 17,21\% siendo muy similar a la observada en las provincias de Chupaca, Huancayo y Concepción.

TABLA 3 Prueba HSD de Tukey, población de insectos por provincia

\begin{tabular}{cccc}
\hline Provincia & N & \multicolumn{2}{c}{ Subconjunto para alfa $=0,05$} \\
& & 2 & 1 \\
\hline Chupaca & 12 & 2070,33 & \\
Huancayo & 12 & 3042,58 & 3042,58 \\
Concepción & 12 & 3460,25 & 3460,25 \\
Jauja & 12 & & 4019,33 \\
\hline \multicolumn{3}{c}{$\mathrm{p}=0,052$}
\end{tabular}

En la Tabla 3, según la prueba de Tukey, muestra que la media poblacional de insectos en S. junceum L. para el valle del Mantaro, existe diferencias estadísticas entre los subconjuntos I y $2(p=0,052)$ y es estadísticamente similar; y según la distribución de la media poblacional forma el subconjunto | siendo estadísticamente igual para el número de individuos observado en Huancayo con 3042,58; Concepción con 3460,25 y Jauja con 4019,33 y el mayor para el valle del Mantaro. En el subconjunto 2 la media poblacional del número de individuos observado en las provincias de Chupaca fue menor con 20,70; Huancayo con 20,42 y Concepción con 34,60. Finalmente existe diferencia estadísticamente significativa para la media poblacional del número de insectos observado en Jauja con relación a Chupaca.

Tabla 4. Población de insectos según familia en $S$. junceum $L$. colectado en cuatro provincias del valle del Mantaro

\begin{tabular}{|c|c|c|c|c|c|c|}
\hline $\mathbf{N}^{*}$ & Fanilia & Oupaca & Huancaye & Consegein & Laja & $\$$ \\
\hline 1 & Baesdat & 7 & 13 & 40 & i & 0,06 \\
\hline 2 & Coenagrionidse & 14 & $\mathbf{3}$ & 12 & 2 & 0,02 \\
\hline 3 & Neshnidae & 15 & 14 & 33 & 10 & 0,05 \\
\hline 4 & Lablladidae & 30 & 26 & 18 & 15 & 0,05 \\
\hline 5 & Bathidae & 45 & 4 & B & 18 & 0,05 \\
\hline 6 & Cnobeencidue & 15 & 38 & 24 & 26 & 0,07 \\
\hline 7 & Gntidae & 40 & 212 & 46 & 33 & 0,22 \\
\hline b & Acrididae & 128 & 239 & $26 \mathrm{~s}$ & 379 & 0,67 \\
\hline 9 & Labidae & 20 & 45 & 8 & 17 & 0,06 \\
\hline 10 & Labituridae & 25 & 3 & 4 & 4 & 0,02 \\
\hline 11 & Forficulidae & 10 & 4 & 11 & 4 & 0,02 \\
\hline 12 & Procidae & 554 & 102 & 45 & 3 & 0,47 \\
\hline $\begin{array}{l}13 \\
14\end{array}$ & $\begin{array}{l}\text { Thrigidae } \\
\text { Pysfidae }\end{array}$ & $\begin{array}{l}5303 \\
812\end{array}$ & $\begin{array}{c}13504 \\
250\end{array}$ & $\begin{array}{l}2072 \\
356\end{array}$ & $\begin{array}{c}20463 \\
129\end{array}$ & $\begin{array}{l}30,57 \\
1,04\end{array}$ \\
\hline 15 & Mphidilae & 8009 & 11538 & 2511 & 17960 & 4220 \\
\hline 16 & Margavedidae & 1744 & 1960 & 225 & 347 & 2,30 \\
\hline 17 & Cicudidne & 65 & 24 & 121 & 61 & $0,1 \mathrm{a}$ \\
\hline 1s & Cloadetilae & 366 & 267 & 450 & 563 & 127 \\
\hline 19 & Anhoconidat & 164 & 465 & 251 & 667 & 1,02 \\
\hline 20 & Mindae & 22 & 48 & 93 & 13 & 0,10 \\
\hline 21 & Lygeidae & 13 & 11 & 13 & 9 & 0,03 \\
\hline 22 & Coneidie & 31 & $n$ & 45 & 111 & 0,14 \\
\hline 23 & Hemerobida & 7 & 11 & 12 & $\mathbf{3}$ & 0,02 \\
\hline 24 & Ongsoptidae & $2 \mathrm{~s}$ & 15 & 0 & 2 & 0,003 \\
\hline 25 & Carabidae & 225 & 14 & 128 & 115 & 0,32 \\
\hline 26 & Saphybidse & 11 & 31 & 134 & 139 & 0.21 \\
\hline 27 & Somabseidue & 7 & 49 & $\boldsymbol{2}$ & 38 & 0,03 \\
\hline 28 & Bosthehisate & 40 & 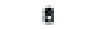 & 10 & 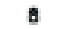 & 0,04 \\
\hline 29 & Melyidae & 216 & 462 & 5 & 325 & 0,70 \\
\hline 30 & Cocoindlide & 41 & 480 & 304 & 354 & $0,7 \mathrm{a}$ \\
\hline $\begin{array}{l}31 \\
x 2\end{array}$ & $\begin{array}{l}\text { Conlophidae } \\
\text { Meloidae }\end{array}$ & $\begin{array}{c}7 \\
61\end{array}$ & $\frac{0}{26}$ & $\stackrel{0}{2}$ & $\stackrel{2}{35}$ & $\begin{array}{l}0,01 \\
0,10\end{array}$ \\
\hline 33 & Ongomelidse & 739 & 500 & 614 & 535 & 1,58 \\
\hline 34 & Curculonidae & 31 & 30 & 17 & 99 & 0,12 \\
\hline 25 & Tipuldae & 165 & 143 & 139 & 181 & 0,42 \\
\hline 36 & Cilbidse & 166 & 364 & 205 & 95 & 0,57 \\
\hline 37 & Ormenemida & 13 & 20 & 25 & 16 & 0,05 \\
\hline 38 & Sinulidae & $\boldsymbol{n}$ & 11 & 38 & 10 & 0,05 \\
\hline 39 & Babionidae & 150 & 359 & 20 & 36 & 0,41 \\
\hline
\end{tabular}




\begin{tabular}{|c|c|c|c|c|c|c|}
\hline $\mathbb{N}^{*}$ & Famia & Chuprasa & Hancaso & Concipeín & hova & 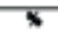 \\
\hline $\begin{array}{l}40 \\
41\end{array}$ & $\begin{array}{l}\text { Cecibonyidae } \\
\text { Mycetophildae }\end{array}$ & $\begin{array}{l}17 \\
68\end{array}$ & $\begin{array}{l}18 \\
8\end{array}$ & ? & 19 & $\begin{array}{l}0,04 \\
0,12\end{array}$ \\
\hline 42 & Xxpphagdae & 17 & 11 & 1 & 0 & 0,02 \\
\hline 43 & Molidae & 109 & 89 & 16 & 17 & 0,15 \\
\hline 44 & Bombytibe & 26 & 7 & 9 & 20 & 0,04 \\
\hline 45 & Enpididae & 36 & 2 & 0 & 7 & 0,03 \\
\hline 46 & Pponculidx & 305 & 400 & 262 & 179 & 0,70 \\
\hline 47 & Spptidise & 361 & 50 & 321 & 166 & 0,9 \\
\hline 48 & Lonchaeidse & is & 4 & 10 & 21 & 0,03 \\
\hline 49 & Cetdise & 62 & 6 & 15 & 23 & 0,07 \\
\hline 50 & Tephetsae & 279 & 0 & 10 & 19 & 0,20 \\
\hline 51 & Aosmydise & 227 & 766 & 400 & 474 & 1,69 \\
\hline 52 & Adelide & 0 & 0 & 0 & 2 & 0,01 \\
\hline 53 & Droscphildse & 4 & 13 & 9 & 23 & 0,09 \\
\hline 54 & CWorepidae & $\mathbf{z}$ & 0 & 1 & 10 & 0,03 \\
\hline 55 & Sectophagdae & 203 & $m$ & 193 & 144 & 0,50 \\
\hline 56 & Mhomyidae & 157 & 242 & 82 & 194 & 0,45 \\
\hline 57 & Muscidise & 475 & 509 & 609 & 570 & 1,43 \\
\hline 58 & Callphoridae & 155 & 392 & 200 & 149 & 0,56 \\
\hline 59 & Sunocphagide & 105 & 178 & 134 & 50 & 0,31 \\
\hline 80 & Tachindate & 95 & 127 & 172 & 235 & 0,42 \\
\hline 61 & Gelechidse & $\pi$ & 31 & $n$ & 7 & 0,12 \\
\hline 62 & Pyoldae & 13 & 20 & 18 & 9 & 0,04 \\
\hline 63 & Pretdan & 25 & 20 & 8 & 6 & 0,04 \\
\hline os & Nymphalidxe & 15 & 2 & 10 & 25 & 0,05 \\
\hline 65 & Noebietae & 24 & 11 & 12 & 7 & 0,04 \\
\hline 66 & Chalcididae & 67 & 8 & 8 & is & 0,06 \\
\hline 6 & Pheromalidue & 31 & 15 & 319 & $2 z$ & 0,26 \\
\hline 68 & Excotidae & 124 & 158 & 250 & 37 & 0,39 \\
\hline 69 & khneumonidas & 167 & 93 & 363 & 113 & 0,47 \\
\hline 70 & Braconidae & 439 & 39 & 159 & 1228 & 1,47 \\
\hline 71 & Fomicidae & 963 & 909 & 752 & 995 & 2,41 \\
\hline$n$ & Pomplidae & 18 & 54 & 79 & 16 & 0,11 \\
\hline 73 & Uerpidae & 33 & 46 & 30 & 20 & 0,09 \\
\hline 74 & Sphecidae & 10 & 62 & 8 & 16 & 0,06 \\
\hline 75 & Mindenidue & 6 & 6 & 4 & 21 & 0,02 \\
\hline 76 & Ahophoridae & 13 & 40 & 13 & 27 & 0,06 \\
\hline$n$ & hidse & 37 & 106 & $n$ & 45 & 0,17 \\
\hline 78 & Halietsue & 7 & 66 & 5 & 3 & 0.06 \\
\hline & ToE & 28544 & उह5ा & $4 \sqrt{523}$ & 46235 & 1000 \\
\hline
\end{tabular}

Analizando la Tabla 4, la distribución el número de insectos por familias en las provincias de Chupaca, Huancayo, Concepción y Jauja, no tuvieron una distribución normal, según la prueba Kolmogorov Smirnov (Sig = 0,000; Z $\alpha=0,05)$. Se observa que las familias Aphididae y Thripidae, son las más representativas y de mayor abundancia con $42,20 \%$ y $30,67 \%$ respectivamente. Mientras que las familias Margaroridae con 2,30\%; Cicadellidae con I,27\%; Psyllidae con 1,04\%; Anthocoridae con 1,02\%; Chrysomelidae con 1,58\%; Agromyzidae con 1,69\%; Muscidae con 1,43\%; Braconidae con 1,47\% y Formicidae con 2,41\% su presencia está considerada como plagas potenciales como Margaroridae; mientras que los cicadélidos la mosca minadora son ocasionales. Destaca la presencia de múscidos y formícidos atraído por la melaza liberada por los pulgones. Las hormigas cumplen un rol de mutualismo y se alimentan de la melaza y ahuyenta a enemigos naturales de los pulgones como las moscas sirfidas. Sin embargo, destaca la presencia individuos de la familia Braconidae, que son controladores biológicos de pulgones, y en particular habiendo identificado al parasitoide nativo del género Pauesia sp., cuya eficiencia de control biológico es baja a los pulgones que ataca a $\mathrm{S}$. junceum L. Se observó que el número de individuos de familias observadas no fue representativo, siendo con un valor menor al uno por ciento en las cuatro provincias para el valle del Mantaro.

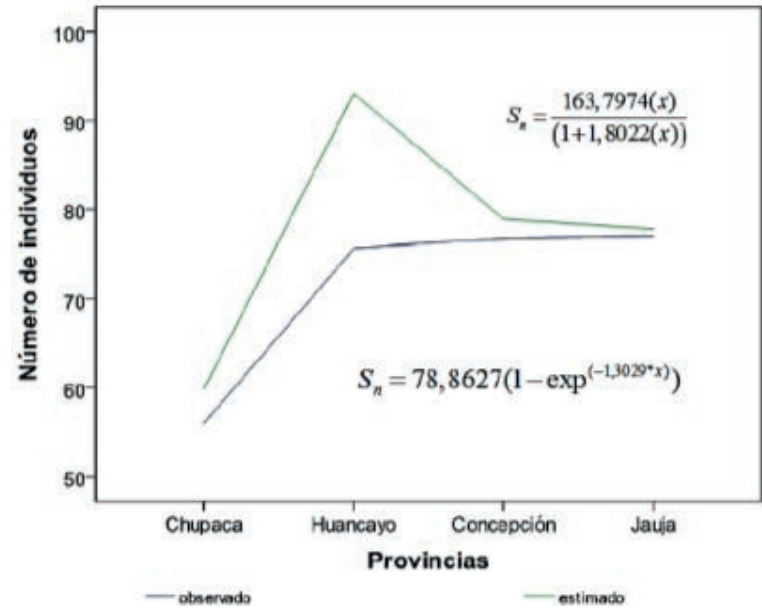

Figura 6. Curva de rarefacción población insectos en S. junceum L. por provincia muestreada valle del Mantaro.

\section{Eficiencia muestral:}

$$
\begin{gathered}
E A=\frac{\text { Número total de individuos observados }}{\text { Número total de individuos estimados }} * 100 \\
E A=\frac{285,42}{309,55} * 100=92,20 \%
\end{gathered}
$$

La eficiencia muestral fue mayor a $80 \%$, significando casi todas las familias presente el área de estudio estuvieron bien distribuidas en las cuatro provincias: Chupaca, Huancayo, Concepción y Jauja.

Para el ajuste de las curvas asintóticas y no asintóticas, fue aplicada la referida por Soberón, J. \& Llorente, J. ( 1993); Gonzales J.A, et al. (20 I 0) y Moreno, C. (200 I). Para el ajuste de las curvas asintóticas y no asintóticas, fue aplicada la referida por Soberón, J. \& Llorente, J. (1993); Gonzales J.A, et al. (20 I0) y Moreno, C. (200 I).

\section{Ecuación no asintótica:}

$$
S_{n}=\frac{a x}{(1+b x)} \quad S_{n}=\frac{163,7974(x)}{(1+1,8022(x))}
$$

Ecuación asintótica ajustada, fue aplicada por Clench (1979), referido por Soberón, J. \& Llorente, J. (1993); Gonzales J.A et al., (2010) en curva asintótica. (Figura 6).

$$
S_{n}=a\left(1-\exp ^{\left(-b^{*} x\right)}\right) S_{n}=78,8627\left(1-\exp ^{\left(-1,3029^{*} x\right)}\right)
$$

\begin{tabular}{|c|c|c|c|c|}
\hline Modelo & CME & $\begin{array}{c}\text { Error } \\
\text { estándar }\end{array}$ & $\mathbf{R}$ & $\mathrm{AlC}$ \\
\hline $\begin{array}{l}\text { Clench } \\
\text { Exponencial } \\
\text { negativo }\end{array}$ & $\begin{array}{l}37,574 \\
12,949\end{array}$ & $\begin{array}{l}4,334 \\
2,545\end{array}$ & $\begin{array}{l}88,29 \\
95,97\end{array}$ & $\begin{array}{l}26,31 \\
22,05\end{array}$ \\
\hline
\end{tabular}

TABLA 5 Indicadores estadísticos para la selección del modelo con mayor bondad de ajuste de curva de rarefacción. 
CME: cuadrado medio del error, $\mathrm{R}^{2}$ coeficiente de determinación, criterio de información de Akaike de los modelos evaluados el que presenta mejor ajuste fue el exponencial negativo con un coeficiente de determinación de 95,97\% y 22,05 \% (AIC).(Tabla 5).

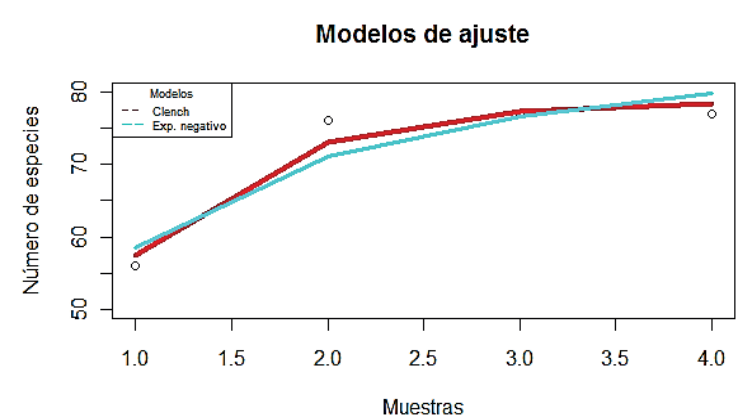

Figura 7. Modelo de la curva de rarefacción de la distribución de insectos en S. junceum L. en el valle del Mantaro.

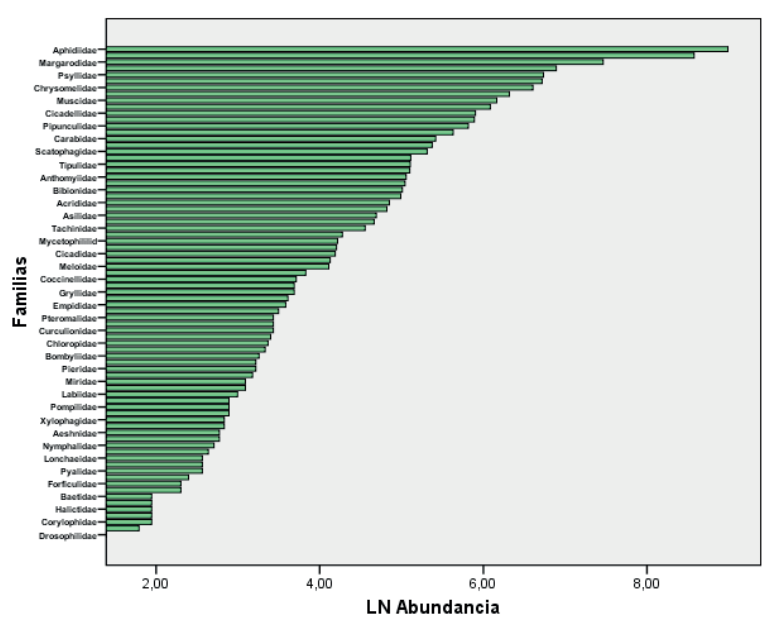

Figura 8. Curva logarítmica de abundancia de insectos en Chupaca

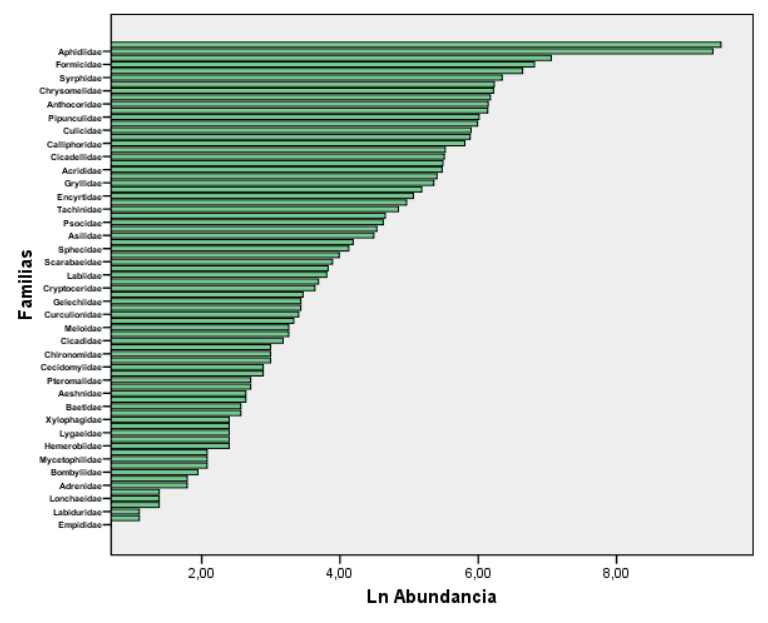

Figura 9. Curva logarítmica de abundancia de insectos en Huancayo

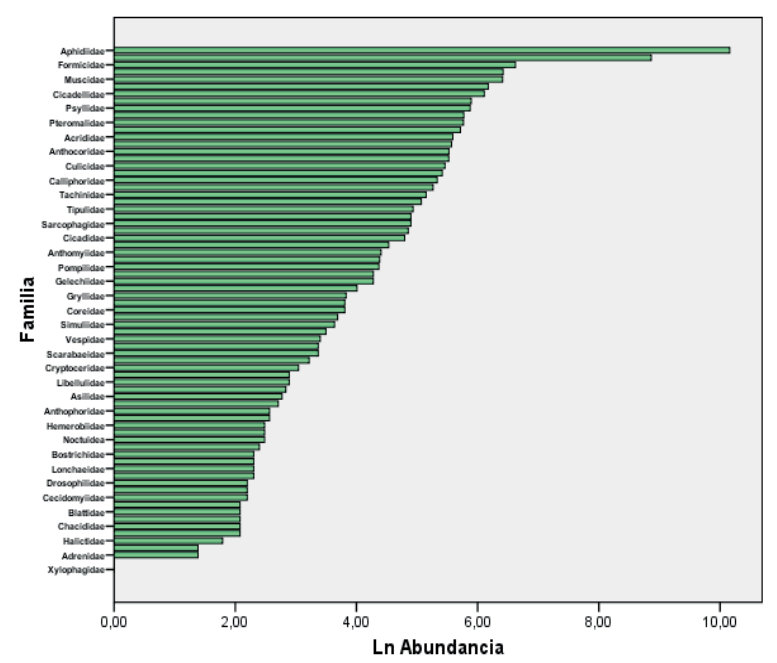

Figura 10. Curva logarítmica de abundancia de insectos en Concepción

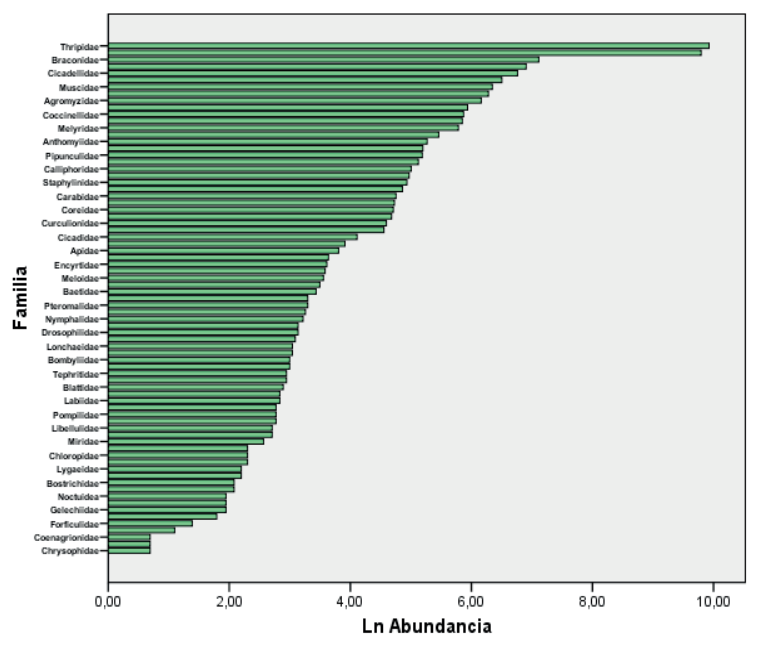

Figura I I. Curva logarítmica de abundancia de insectos en Jauja

Las Figuras 8, 9, 10 y 11 , representan la curva logarítmica ajustada de la abundancia de la población de los insectos observado en S. junceum L. en cada provincia muestreada en el valle del Mantaro. La población por el número de individuos representado por las curvas tiene una tendencia similar para Chupaca, Huancayo, Concepción y Jauja. Se distingue la dominancia de familias tienen mayor número de individuos registrado, como es la Aphididae, Thripidae. (Magurran, 1988); (Krebs, 1978) y (Krebs, 1989). 
Estimación de la diversidad de insectos en S. junceum

Tabla 6. Índices de diversidad estimado, año 2010

\begin{tabular}{ccccccc}
\hline Mes & Familias & Individuos & Simpson & Shannon & Margalef & $\begin{array}{c}\text { Berger- } \\
\text { Parker }\end{array}$ \\
\hline Enero & 47,25 & 4718,75 & 0,67 & 1,77 & 5,50 & 0,45 \\
Febrero & 50,50 & 3502,25 & 0,74 & 2,07 & 6,16 & 0,40 \\
Marzo & 51,25 & 3086,25 & 0,69 & 1,82 & 6,26 & 0,48 \\
Abril & 55,75 & 2398,25 & 0,65 & 1,85 & 7,35 & 0,55 \\
Mayo & 57,50 & 1856,00 & 0,69 & 1,96 & 7,57 & 0,50 \\
Junio & 59,00 & 1791,00 & 0,70 & 2,04 & 8,01 & 0,47 \\
Julio & 55,50 & 1836,00 & 0,69 & 1,97 & 7,28 & 0,48 \\
Agosto & 59,50 & 1259,50 & 0,69 & 1,96 & 8,20 & 0,49 \\
Setiembre & 60,25 & 3983,00 & 0,70 & 1,98 & 7,19 & 0,46 \\
Octubre & 64,50 & 3556,75 & 0,72 & 2,04 & 7,89 & 0,42 \\
Noviembre & 60,75 & 4453,75 & 0,72 & 2,00 & 7,18 & 0,41 \\
Diciembre & 61,00 & 5336,25 & 0,70 & 1,86 & 7,04 & 0,43 \\
\hline $\mathbb{1}$ & 56,90 & 3148,15 & 0,70 & 1,94 & 7,13 & 0,46 \\
\hline S & 5,06 & 1325,10 & 0,02 & 0,09 & 0,81 & 0,04 \\
\hline CV & 0,09 & 0,42 & 0,03 & 0,05 & 0,11 & 0,09 \\
\hline
\end{tabular}

En la Tabla 6, muestra el resultado de la estimación de los índices de diversidad por meses año 2010, de la población de insectos muestreado en Chupaca, Huancayo, Concepción y Jauja. El número promedio mensual de familias observadas varió de 47,25 en el mes de enero a 64,50 en el mes de octubre. El número familias tuvo una media 56,90 para el valle del Mantaro.

Con relación al promedio mensual del número de individuos, también varió de 1259,50 en agosto a 5336,25 en diciembre. Este resultado muestra que una población de insectos es más abundante y diversa cuando las relaciones existentes en el ecosistema (factores bióticos y abióticos) son favorables y determinaron la abundancia y guarda relación con el esfuerzo de muestreo obtenido en la Figura 7.

Tabla 7. Anova prueba de comparación de medias índices diversidad provincias

\begin{tabular}{|c|c|c|c|c|c|c|}
\hline \multicolumn{2}{|l|}{ İndice } & $\mathrm{GL}$ & sc & $\mathrm{CM}$ & $\mathrm{F}$ & Sig. \\
\hline \multirow[t]{3}{*}{ Shannon } & \multirow{3}{*}{$\begin{array}{l}\text { Inter- } \\
\text { grupos } \\
\text { Intra- } \\
\text { grupos } \\
\text { Total }\end{array}$} & 3 & 3,793 & 1,264 & 31,003 & 0,000 \\
\hline & & 44 & 1,794 & 0,041 & & \\
\hline & & 47 & 5,587 & & & \\
\hline \multirow[t]{3}{*}{ Simpson } & \multirow{3}{*}{$\begin{array}{l}\text { Inter- } \\
\text { grupos } \\
\text { Intra- } \\
\text { grupos } \\
\text { Total }\end{array}$} & 3 & 1,942 & 0,647 & 249,010 & 0,000 \\
\hline & & 44 & 0,114 & 0,003 & & \\
\hline & & 47 & 2,056 & & & \\
\hline \multirow[t]{3}{*}{ Margalef } & \multirow{3}{*}{$\begin{array}{l}\text { Inter- } \\
\text { grupos } \\
\text { Intra- } \\
\text { grupos } \\
\text { Total }\end{array}$} & 3 & 3,947 & 1,316 & 1,473 & 0,235 \\
\hline & & 44 & 39,297 & 0,893 & & \\
\hline & & 47 & 43,243 & & & \\
\hline \multirow[t]{3}{*}{ BergerParker } & \multirow{3}{*}{$\begin{array}{l}\text { Inter- } \\
\text { grupos } \\
\text { Intra- } \\
\text { grupos } \\
\text { Total }\end{array}$} & 3 & 0,453 & 0,151 & 32,019 & 0,000 \\
\hline & & 44 & 0,207 & 0,005 & & \\
\hline & & 47 & 0,660 & & & \\
\hline
\end{tabular}

En la Tabla 7, se observa el análisis de varianza de la comparación de medias de los índices de diversidad estimado para las provincias de Chupaca, Huancayo, Concepción y Jauja, de los insectos que hospeda S. junceum L., en donde se aprecia que para las medias de los índices de dominancia, Shannon, Simpson, y Berger Parker existen diferencias estadísticas significativas $(p=0,000)$; para cada índice, y siendo uno de ellos el mayor. Mientras que la media del índice de Margalef estimado para la provincia de Chupaca, Huancayo, Concepción y Jauja, no existe diferencia estadística significativa $(p=0,235)$.
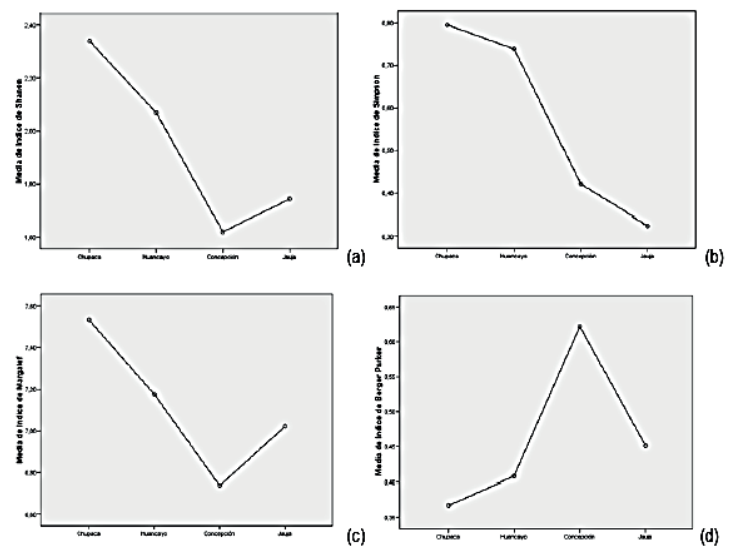

Figura I2. Gráfico de la media de los índices de Shannon -Wiener (a); Simpson (b); Margalef (c) y Berger Parker (d).

En la Figura 12, las medias de los índices estimados de la población en S. junceum L. señala que la figura de Shannon-Wiener (a), Simpson (b), Margalef (c) y Berger Parker (e) existen diferencias de los valores medios estimado, siendo uno de ellos mayor del resto para caso de los índices estimados. Mientras que para el índice de Margalef (d), los valores de las medias son iguales estadísticamente según la prueba Tukey aplicada.

Tabla 8 Prueba " $t$ " de Hutcheson (1970), índice de Shannon - Wiener por provincias del valle del Mantaro

\begin{tabular}{|c|c|c|c|}
\hline Chupaca & Huancayo & Huancayo & Concepción \\
\hline $\mathrm{S}=77$ & $S=74$ & $S=74$ & $S=73$ \\
\hline$H^{\prime}=2,6142$ & $H^{\prime}=2,1312$ & $H^{\prime}=2,1312$ & $H^{\prime}=1,6579$ \\
\hline Var $=0,00010844$ & $\operatorname{Var}=8,241 \mathrm{E}-05$ & Varianza $=8,241 E-05$ & Varianza $=8,4839 E-05$ \\
\hline \multicolumn{2}{|c|}{$t=34,957$} & \multicolumn{2}{|c|}{$t=36,6$} \\
\hline \multicolumn{2}{|c|}{$d f=55243$} & \multicolumn{2}{|c|}{$d=77841$} \\
\hline \multicolumn{2}{|c|}{ Sign $(0,05)=7,90225 E-265$} & \multicolumn{2}{|c|}{$\operatorname{Sign}(0,05)=8,6942 E-29$} \\
\hline Concepción & Jauja & Jauja & Chupaca \\
\hline$S=73$ & $\mathrm{~S}=\pi$ & $\mathrm{s}=77$ & $\mathrm{~S}=77$ \\
\hline$H^{\prime}=1,6579$ & $H^{\prime}=1,7297$ & $H^{\prime}=1,7297$ & $H^{\prime}=2,6142$ \\
\hline Var $=8,4839 E-05$ & Var= $5,940 E-05$ & Var $=0,59402 E-05$ & Var $=0,00010844$ \\
\hline \multicolumn{2}{|c|}{$t=-5,9731$} & \multicolumn{2}{|c|}{$t=68,274$} \\
\hline \multicolumn{2}{|c|}{$d=84404$} & \multicolumn{2}{|c|}{$d t=51550$} \\
\hline \multicolumn{2}{|c|}{$\operatorname{Sign}(0,05)=2,3374 E-09$} & \multicolumn{2}{|c|}{$\operatorname{Sign}(0,05)=0$} \\
\hline
\end{tabular}

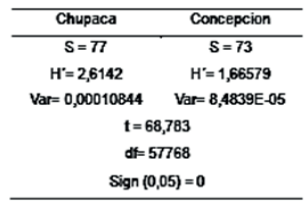

En la Tabla 8, el resultado de la aplicación de test de la prueba de t de Hutcheson (1970), para comparar el índice de diversidad de Shannon - Wiener para cada dos provincias muestreadas. Los resultados indican que para los casos evaluados en la provincia de Chupaca y con cada provincia par (Huancayo, Concepción y Jauja), existe diferencia estadística significativa $(p=0,000)$ sien- 
do mayor el valor del índice Shannon-Wiener para los casos observados de los insectos. Sin embargo, cuando se compara los insectos observados en la provincia de Huancayo, con la provincia de Concepción y Jauja, existe diferencia estadística significativa, por la media del mayor valor del índice de Shannon - Wiener estimado. Además mostraron diferencias estadísticas significativas de la estimación de la diversidad de población de insectos en la "retama".

Factores climáticos que influyen en la diversidad de insectos en S. junceum L. en el valle del Mantaro

Tabla 9 Promedio mensual del clima valle del Mantaro, año 2010.

\begin{tabular}{cccccc}
\hline Mesaño 2010 & $\mathrm{N}^{\circ}$ insectos & $\begin{array}{c}\text { Temperatura } \\
\left({ }^{\circ} \mathrm{C}\right)\end{array}$ & $\begin{array}{c}\text { Precipitación } \\
(\mathrm{mm})\end{array}$ & $\begin{array}{c}\text { Humedad } \\
\text { relativa }(\%)\end{array}$ & $\begin{array}{c}\text { Horas sol } \\
(\mathrm{hs})\end{array}$ \\
\hline Enero & 18875 & 13,46 & 145,34 & 71,80 & 138,37 \\
Febrero & 14009 & 13,83 & 103,81 & 71,60 & 122,60 \\
Marzo & 12345 & 13,74 & 82,78 & 70,40 & 157,00 \\
Abril & 9593 & 13,08 & 42,11 & 66,60 & 184,63 \\
Mayo & 7424 & 12,33 & 2,84 & 60,60 & 248,80 \\
Junio & 7164 & 11,10 & 7,20 & 61,40 & 241,17 \\
Julio & 7344 & 10,75 & 5,04 & 59,80 & 300,80 \\
Agosto & 5038 & 11,20 & 3,18 & 61,00 & 274,85 \\
Setiembre & 15932 & 12,68 & 16,76 & 60,60 & 207,30 \\
Octubre & 14227 & 13,43 & 58,18 & 63,60 & 183,60 \\
Noviembre & 17815 & 13,50 & 47,60 & 63,20 & 175,37 \\
Diciembre & 21345 & 13,07 & 124,70 & 68,20 & 125,83 \\
\hline Media & 12592,58 & 12,68 & 53,30 & 64,90 & 196,69 \\
\hline S & 5300,41 & 1,09 & 50,47 & 4,59 & 58,79 \\
\hline CV & 0,42 & 0,09 & 0,95 & 0,07 & 0,30 \\
\hline
\end{tabular}

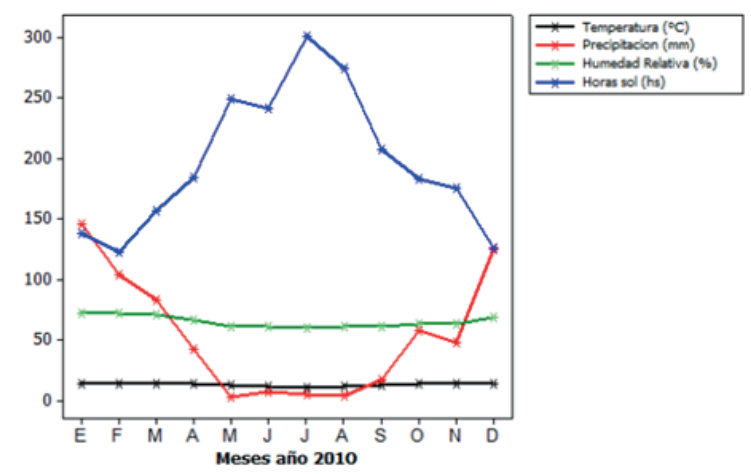

Figura I3. Fluctuación del clima en el valle del Mantaro año 2010.

Las variables climáticas observado en el valle del Mantaro, durante el periodo evaluado, fue para la temperatura con valor de $1 \mathrm{I}$ a $13^{\circ} \mathrm{C}$, y se aprecia que el coeficiente de variación fue muy próximo al uno por ciento, significando que los rangos no fueron muy extremos y favoreció a la presencia de insectos en S. junceum L.

Sin embargo, la precipitación adquirió valores de 3 a 145 mm, mostrando en los meses de mayo a setiembre escasa precipitación, y hubo un incremento significativo en los meses de enero a marzo y luego de octubre a diciembre durante el periodo observado. Esta ocurrencia de la precipitación obtuvo un coeficiente de variación de 95\%, indicando que fue muy disperso.

La humedad relativa fue de $61 \%$ a $72 \%$, y obtuvo un coeficiente de variación de 7 por ciento, siendo un valor que estuvo relacionado con la temperatura, pluviosidad y horas sol.

Las horas sol vario de 123 a un máximo de 275 respectivamente; esta variable fue observada en mayor proporción durante los meses de menor precipitación y fue menor en los meses de mayor pluviosidad para el valle del Mantaro. (Tabla 9 y Figura 13).

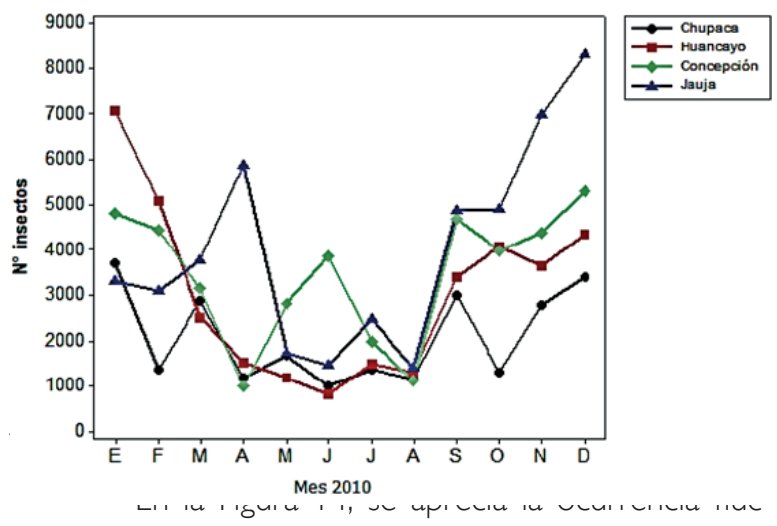

tuación poblacional de los insectos observado en S. junceum L; y en el valle del Mantaro, en los meses de mayor precipitación, humedad relativa y horas sol la población de insectos se incrementó; pero mostraron valores poblacionales bajos, cuando los factores climáticos tuvieron valores bajos. Esta tendencia fue muy similar la observada en las provincias de Chupaca, Huancayo, Concepción y Jauja respectivamente.

Tabla 10. Correlación del número de insectos y el clima en el valle del Mantaro.

\begin{tabular}{clcccc}
\hline & & $\begin{array}{c}N^{\circ} \text { de } \\
\text { insectos }\end{array}$ & $\begin{array}{c}\text { Precipitación } \\
(\mathrm{mm})\end{array}$ & $\begin{array}{c}\text { Humedad } \\
\text { relativa (\%) }\end{array}$ & $\begin{array}{c}\text { Horas sol } \\
\text { (hs) }\end{array}$ \\
\hline Correlación de & $N^{\circ}$ de insectos & 1,000 & 0,782 & 0,551 & $-0,814$ \\
Pearson & Precipitación (mm) & 0,782 & 1,000 & 0,916 & $-0,893$ \\
& Humedadrelativa & 0,551 & 0,916 & 1,000 & $-0,869$ \\
& $(\%)$ & & & & \\
& Horassol (hs) & $-0,814$ & $-0,893$ & $-0,869$ & 1,000 \\
\hline Sig. (unilateral) & $N^{\circ}$ de insectos &. & 0,001 & 0,032 & 0,001 \\
& Precipitación (mm) & 0,001 &. & 0,000 & 0,000 \\
& Humedadrelativa & 0,032 & 0,000 &. & 0,000 \\
& $(\%)$ & & & \\
& Horassol (hs) & 0,001 & 0,000 & 0,000 &. \\
\hline
\end{tabular}

La Tabla 10, muestra la correlación de Pearson de las variables del Numero de insectos y las variables del clima, como la precipitación ( $\mathrm{mm}$ ), humedad relativa (\%) y horas (hs), cuyo valor a un nivel de significancia $(\alpha=0,05)$ con correlaciones mayores $0,55 \mathrm{l}$. La correlación fueron significativa presentar valores menor o igual a ( $p$ $\leq$ 0,05); antes de ser sometida al análisis con regresión múltiple de las variable independientes de precipitación $(\mathrm{mm})$, humedad relativa (\%) y horas (hs) con la variable 
respuesta número de insectos.

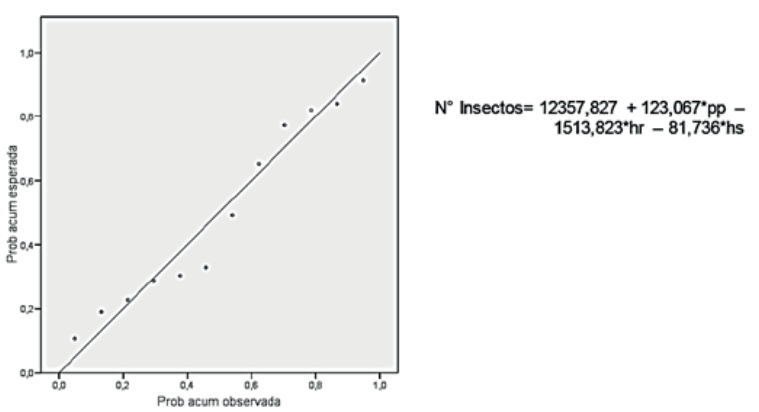

Figura I5. Regresión múltiple de la población de insectos en S. junceum L. y el clima del valle del Mantaro.

En la Figura 15, se observa la ecuación de regresión lineal múltiple ajustada, siendo $\mathrm{N}^{0}$ Insectos $(Y)=$ $12357.827+123.067 * p p-1513.823 * h r-81.736 * h s ;$ donde $\beta o=12357,827$ representa el valor medio de número de insectos en la "retama", sin considerar la influencia de la precipitación, humedad relativa y horas sol (factores climáticos). Mientras que $\beta \mid=+123.067^{*} p p$ representa que si aumenta la precipitación, manteniendo constante la humedad relativa y horas sol, el número de insectos en la "retama" aumenta en 123.067 individuos. Sin embargo $\beta 2=-1513.823^{*} \mathrm{hr}$ representa si aumenta la humedad relativa, manteniendo constante la precipitación y horas sol, el número de insectos en la "retama" disminuye en -1513.823 individuos. Mientras que con $\beta 3=-81.736$ *hs representa si aumenta la horas sol, manteniendo constante la precipitación y humedad relativa, el número de insectos en la "retama", disminuye en - 81.736 individuos.

Los factores climáticos que tuvo mayor influencia en la población de insectos en la "retama", fue la humedad relativa, luego la precipitación y finalmente la humedad relativa, guardando relación al valor de los coeficientes estimado en la regresión lineal múltiple estimada de los insectos evaluado en las provincias de Chupa-

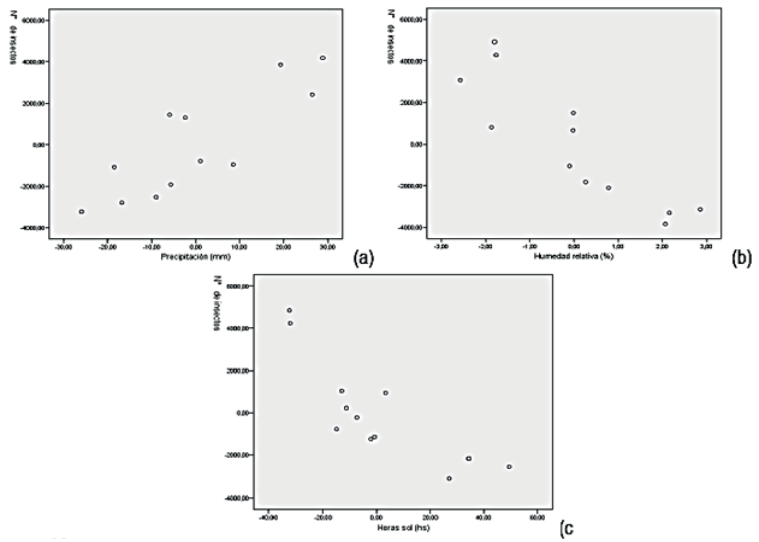

Figura 16. Gráfico regresión parcial de la población de insectos en S. junceum L. y los factores climáticos en el valle del Mantaro. Precipitación (a). Humedad relativa (b). Horas sol (c).
En la Figura 16, se aprecia la referencia a las posibles desviaciones de los datos desde el modelo lineal obtenido. Son diagramas de dispersión de los residuos de las variables predictivas de la precipitación (a), humedad relativa (b) y horas sol (c), y los residuos de la variable respuesta dependiente número de insectos cuando se regresan ambas por separado sobre las restantes variables independientes.

Los resultados muestran que la precipitación (a) y el número de insectos, tras eliminar el efecto del resto de variables independientes, es lineal y positiva. Mientras que la humedad relativa (b) y las horas sol (c) y el número de insectos, tras eliminar el efecto del resto de variables independientes, es para cada variable predictor es lineal y negativa.

Tabla I I. Análisis de varianza de la regresión múltiple

\begin{tabular}{cccccc}
\hline FV & GL & SC & CM & F & Sig. \\
\hline Regresión & 3 & 288092055,632 & 96030685,211 & 36,677 & 0,000 \\
Residual & 8 & 20946319,285 & 2618289,911 & & \\
Total & 11 & 309038374,917 & & & \\
\hline \multicolumn{2}{c}{ R=96,60\% } & $R^{2}=93,20 \%$ & Durbin- Watson $=3,281$
\end{tabular}

En la Tabla I I, se aprecia el resultado del análisis de varianza a la regresión múltiple aplicada, con la prueba estadística $F$ a nivel $(\alpha=0,05)$ siendo la variable respuesta el número de insectos, y las variables independientes de precipitación (mm), humedad relativa (\%) y horas (hs), siendo estadísticamente valida la ecuación de regresión ajustada a un nivel de significancia ( $p=0,000$ ).

Tabla I2. Significación estadística de las variables del clima.

\begin{tabular}{lccccc}
\hline \multicolumn{5}{c}{ Coeficientes no estandarizados } & $\begin{array}{c}\text { Coeficientes } \\
\text { estandarizados }\end{array}$ \\
\hline & B & Eror tip. & Beta & $t$ & Sig. \\
\hline Constante & 120357,827 & 18675,302 & & 6,445 & 0,000 \\
Precipitación & 123,067 & 27,604 & 1,172 & 4,458 & 0,002 \\
(mm) & & & & \\
Humedad relativa & $-1513,823$ & 276,076 & $-1,310$ & $-5,483$ & 0,001 \\
$(\%)$ & $-81,736$ & 19,266 & $-0,907$ & $-4,243$ & 0,003 \\
\hline Horassol (hs) & & & & & \\
\hline
\end{tabular}

En la Tabla 12, se observa que los coeficientes para el modelo de regresión lineal múltiple ajustado, con la prueba de $\mathrm{t}(\alpha=0,05)$, tienen las constantes de la precipitación, la humedad relativa y las horas sol un nivel de significación con valores menor a ( $p=0,05)$ siendo estadísticamente válido el modelo de regresión determinado. 


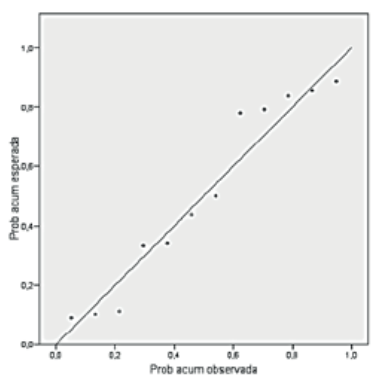

Índice Margalef $=16,580-0,146 * \mathrm{hr}$

Figura I7. Regresión del índice de Margalef y la humedad relativa de la población de insectos en $\mathrm{S}$. junceum $\mathrm{L}$. en el valle del Mantaro.

En la Figura 17, en la regresión (Y) Índice Margalef $=16,580-0,146 * h r$; donde $\beta o=16,580$ representa el valor medio de la respuesta $(Y=$ Índice de Margalef) cuando la variable explicativa vale cero. Sin embargo $\beta|=-0| 46$ representa que disminuye en una unidad cuando la variable explicativa $(X \mid=$ humedad relativa) permanecen constantes. La humedad relativa influye significativamente lineal y negativa en la diversidad estimada por el índice de Margalef según el número de insectos observado que hospeda S. junceum L. en las provincias de Chupaca, Huancayo, Concepción y Jauja del valle del Mantaro.

Tabla I3. Análisis de varianza de la regresión del Índice de Margalef y la humedad relativa (\%).

\begin{tabular}{cccccrr}
\hline FV & GL & SC & CM & F & s \\
\hline Regresión & 1 & 4,903 & & 4,903 & 22,018 & 0, \\
Residual & 10 & 2,227 & & 0,223 & & \\
Total & 11 & 7,130 & & & \\
\hline \multicolumn{5}{c}{$\mathrm{R}=82,90 \%$} & \multicolumn{2}{c}{$\mathrm{R}^{2}=68,80 \%$}
\end{tabular}

La Tabla 13, muestra el análisis de varianza según la prueba F aplicada para determinar que la ecuación de regresión ajustada entre en índice Margalef y la humedad relativa es estadísticamente valida con un nivel de significación ( $p=0,000$ ).

Tabla I4. Significación estadística de la variable humedad relativa y el índice de Margalef.

\begin{tabular}{cccccc}
\hline \multicolumn{5}{c}{ Coeficientes no estandarizados } & $\begin{array}{c}\text { Coeficientes } \\
\text { estandarizados }\end{array}$ \\
\hline & B & Error tip. & Beta & t & Sig. \\
\hline $\begin{array}{c}\text { Constante } \\
\begin{array}{c}\text { Humedad relativa } \\
(\%)\end{array}\end{array}$ & 16,580 & 2,017 & & 8,219 & 0,000 \\
\hline
\end{tabular}

En la Tabla |4, se observa los coeficientes para el modelo de regresión lineal ajustado, con la prueba de t $(\alpha=0,05)$; la constante $(\beta \circ=16,580)$ tiene significación estadística valida $(p=0,000)$; y la humedad relativa $(X|=-0| 46$,$) un nivel de significación (p=0,01$ ) siendo estadísticamente válido el modelo de regresión determinado.
Insectos identificados en S. junceum L. en el valle del Mantaro

Tabla I5. Ordenes, géneros y especies de insectos identificados

\begin{tabular}{|c|c|c|}
\hline Orden & Familia & Especie \\
\hline \multirow{2}{*}{ Odonata } & Aeshniidae & Aeshna sp. \\
\hline & Libellullidae & Anax sp. \\
\hline Dermaptera & Forficulidae & Forficula sp. \\
\hline Orthoptera & Grillydae & $\begin{array}{l}\text { Gryllus } \\
\text { peruvianus }\end{array}$ \\
\hline \multirow{9}{*}{ Hemiptera } & \multirow{3}{*}{ Aphididae } & Aphis cystisorun \\
\hline & & Aphis fabae \\
\hline & & Macrosiphum sp. \\
\hline & Margaroridae & Icerya purchasi \\
\hline & \multirow{4}{*}{ Cicadellidae } & Bergalia sp. \\
\hline & & Empoasca sp. \\
\hline & & Paratanus sp. \\
\hline & & Amblysellus sp. \\
\hline & Anthocoridae & Orius insidiosus \\
\hline \multirow{2}{*}{ Thysanoptera } & \multirow{2}{*}{ Thripidae } & Frankliniella sp. \\
\hline & & Thrips tabaci \\
\hline Neuroptera & Hemerobidae & Hemerobius sp. \\
\hline \multirow{10}{*}{ Coleoptera } & Staphylinidae & Anotylus sp. \\
\hline & Melyridae & Astylus sp. \\
\hline & Carabidae & Harphalus sp. \\
\hline & \multirow[t]{2}{*}{ Coccinellidae } & $\begin{array}{l}\text { Hippodamia } \\
\text { convergens }\end{array}$ \\
\hline & & Eriopis sp. \\
\hline & Meloidae & Epicauta sp. \\
\hline & \multirow{3}{*}{ Chrysomelidae } & Epitrix yanazara \\
\hline & & $\begin{array}{l}\text { Diabrotica } \\
\text { virudula }\end{array}$ \\
\hline & & Galerucinae sp. \\
\hline & Curculionidae & Onaopactus sp. \\
\hline \multirow{15}{*}{ Diptera } & \multirow{2}{*}{ Tipulidae } & Limonia sp. \\
\hline & & Tipula sp. \\
\hline & Pipunculidae & Pipunculus sp \\
\hline & \multirow{2}{*}{ Agromyzidae } & $\begin{array}{l}\text { Liriomyza } \\
\text { huidobrensis }\end{array}$ \\
\hline & & $\begin{array}{l}\text { Liriomyza } \\
\text { patagónica }\end{array}$ \\
\hline & Anthomyiidae & Delia platura \\
\hline & Muscidae & Coenocia sp. \\
\hline & \multirow{3}{*}{ Tachinidae } & Whintemia sp. \\
\hline & & Gonia sp. \\
\hline & & $\begin{array}{l}\text { Prosopochaeta } \\
\text { sp. }\end{array}$ \\
\hline & \multirow{2}{*}{ Syrphidae } & Syrphus sp. \\
\hline & & Scaeva sp. \\
\hline & \multirow{2}{*}{ Lonchaeidae } & Dasiops sp. \\
\hline & & Lonchaea sp. \\
\hline & Scatophagidae & $\begin{array}{l}\text { Scatophaga } \\
\text { estercolera }\end{array}$ \\
\hline Lepidoptera & Gelichiidae & $\begin{array}{l}\text { Eurysaca } \\
\text { melanocapta }\end{array}$ \\
\hline \multirow{9}{*}{ Himenoptera } & \multirow{2}{*}{ Ichneumonidae } & Polistes sp. \\
\hline & & Enicospilus sp. \\
\hline & \multirow{3}{*}{ Braconidae } & Aphidius sp. \\
\hline & & Lysiphlebus sp. \\
\hline & & Praeon sp. \\
\hline & Formicidae & Camponotus sp. \\
\hline & \multirow[b]{2}{*}{ Encyrtidae } & Copidosoma sp. \\
\hline & & $\begin{array}{l}\text { Psyllaephagus } \\
\text { pilossus }\end{array}$ \\
\hline & Aphidae & Aphis mellifera \\
\hline
\end{tabular}




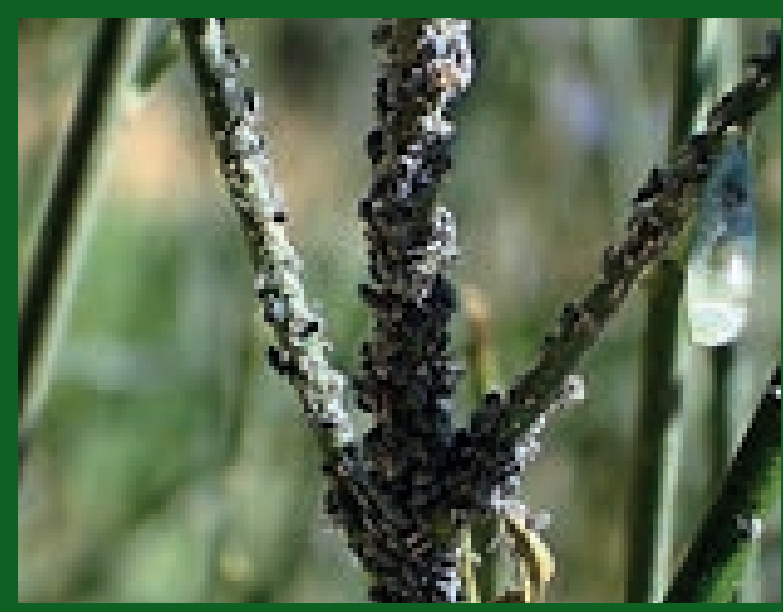

Figura I8. Planta de S. junceum L. atacado por pulgones. El Mantaro Jauja
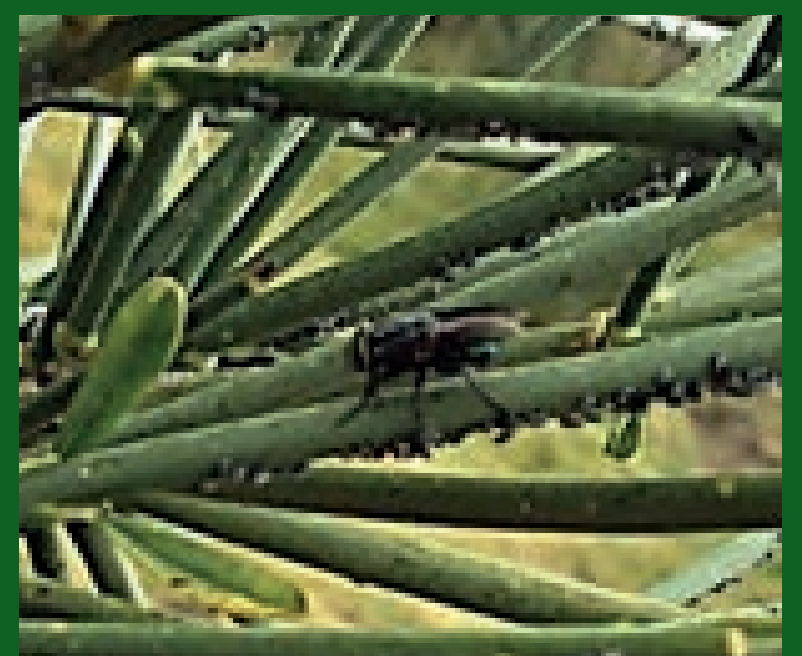

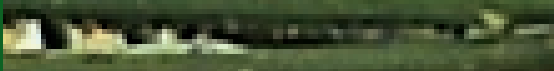

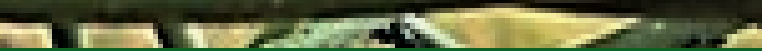

Figura 19. Insecto de la orden Díptera, Familia Calliphoridae

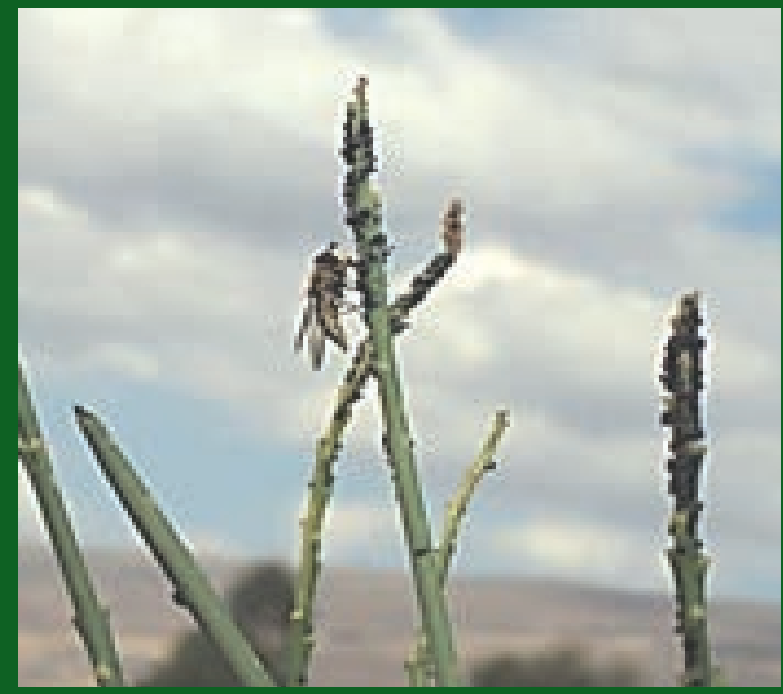

Figura 20. Insecto de la orden Díptera, Familia Syrphidae

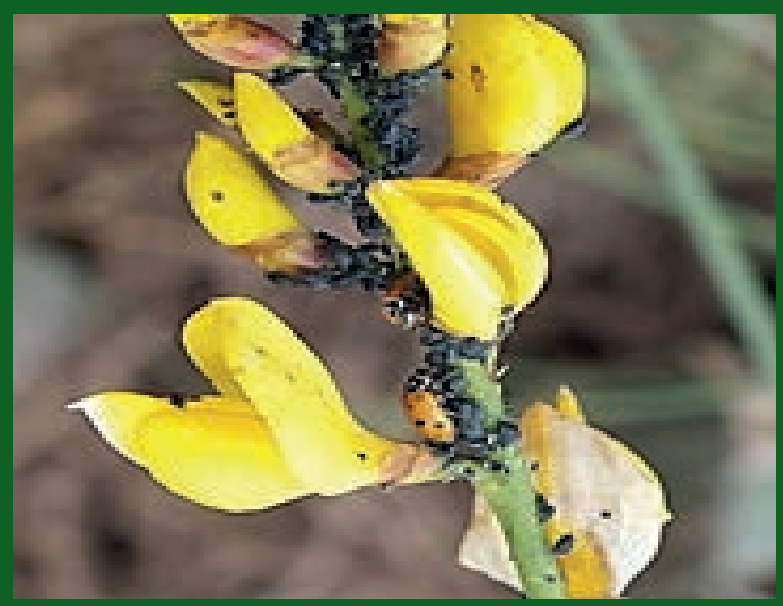

Figura 2I. Hippodamia convergens, alimentándose de pulgones.

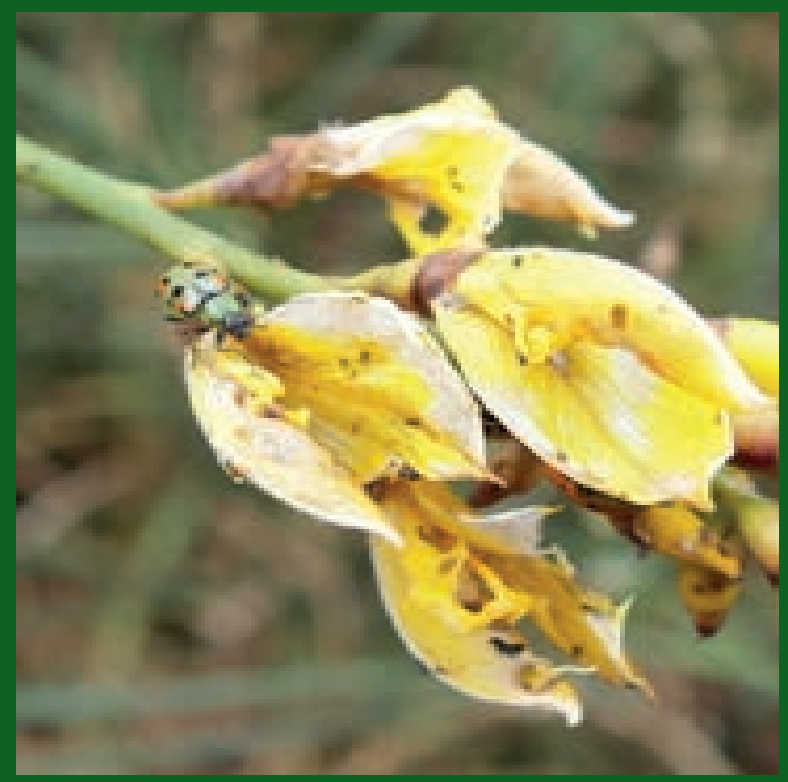

Figura 22. Diabrotica viridula, predatando ninfas de pulgones.

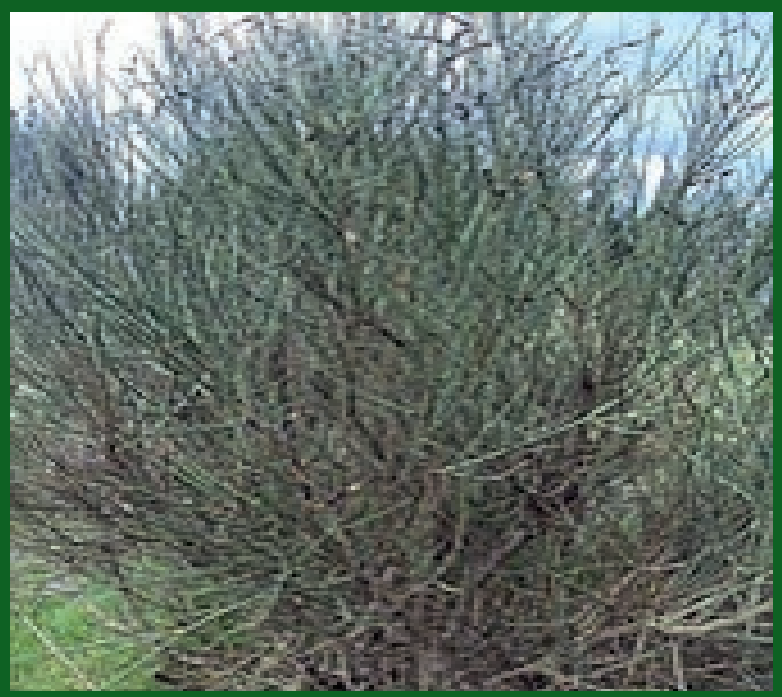

Figura 23. Plantas de "retama" en extinción. 
Composición y estructura de la población de insectos que hospeda S. junceum L. en el valle del Mantaro. El valle del Mantaro comprende territorios de las provincias de Huancayo, Chupaca, Concepción y Jauja, es una zona agrícola importante de la región Junín, y de la sierra peruana, en donde producen diferentes cultivos como Zea mays, Solanum tuberosum, Hordeum vulgare, Triticum aestivum, Cynara scolymus y hortalizas; frutales como Pyrus malus, Prunus persica y Prunus serotina, cuyos productos son destinados al autoconsumo, los mercados locales y a grandes ciudades como Huancayo y Lima, (Ministerio de Agricultura, 2007). Además existen plantaciones forestales valiosas como el Eucalyptus globulus Labill, Schinus molle, Alnus acuminata, Prunus serotina y $S$. junceum L., y dichas especies son hospedantes de una diversidad valiosa e importante de insectos.

Además la "retama", es una leguminosa bien adaptada al suelo y clima andino de la región quechua (Brack y Mendiola, 2004); y crece asociada a con otras especies vegetales, y por esta característica es muy apreciada y explotada en el valle del Mantaro. Sin embargo desde el año 2006, está siendo atacado principalmente por pulgones y como consecuencia está en peligro de extinción.

La estructura poblacional de insectos en $\mathrm{S}$. junceum L., está relacionada a la actividad agronómica y forestal que realiza el poblador del valle del Mantaro, siendo la agricultura la de mayor importancia. Esto ha determinado que hay la presencia significativa de Hemípteros y Thysanopteros, siendo insectos plagas claves como pulgones, y trips que se hospedan en cultivos agrícolas durante la mayor parte del año.

Vilca y Vergara (20II), reportaron que la "retama" hospeda tres importantes especies de pulgones como el Aphis craccivora, Aphis cytisorum (plaga clave); y Aphis fabae respectivamente siendo polífago y cosmopolita en el Callejón de Huaylas (Ancash). Este resultado confirma que los miembros de la familia Aphididae (Insecta: Hemíptera), son insectos de amplia distribución geográfica, principalmente en las regiones templadas; infestan toda clase de vegetales y muchas especies son de importancia económica, por los daños directos que causan durante su alimentación (Klingauf, 1987; Quisenbery \& Xinzhi, 2007) y especialmente por constituir el grupo de insectos más importante en $S$. junceum $L$.

Con relación a la presencia significativa de la orden Thysanoptera, se afirma que S. junceum L. al ser especie que comparte el nicho con otras especies agrícolas y forestales, y por la característica fenológica de floración permanente, constituye un hospedante importante de los "Trips", el cual constituye una plaga secundaria que afecta los pétalos de la flor de dicha especie. También, es importante apreciar la presencia de insectos de la Orden Himenóptera, debido a la atracción floral de la coloración de las flores y duración del periodo fenológico.

Esta característica poblacional de insectos de la familia Aphididae, se debe probablemente por el contenido del alcaloide quinolizidina, siendo un compuesto alelo químico de las Fabáceas presente en plantas de Spartium junceum L. y el Aphis cystisorun en Lupinus como señala Wink y Ludger ( 1990); y esta sustancia es metabolizada sin problema por los pulgones.

Con relación a la presencia significativa de la familia Thripidae, con la especie identificada de Thrips tabaci Lindeman, I888, se confirma que S. junceum L. en el valle del Mantaro, es hospedante principal como reporta Raspudic et al., (2009), para dicha especie arbustiva, y que además son insectos polífagas y cosmopolitas.

Con relación a la presencia de individuos de Familia Formicidae, Orden Himenóptera, se atribuye a que dichos insectos viven en mutualismo con los pulgones, $y$ constituyen plaga principal con tres especies de pulgones identificados como sostiene Vilca y Vergara (20I I); Baltazar, (2007); Rojas y Huamán (2008), respectivamente.

Sin embargo Delfino M. A. y Buffa L. M. (2000), afirman que algunas especies de hormigas predatan 0 defiende de enemigos a los pulgones. Las defienden de predadores y parasitoides, incluso en algunos casos les proveen hábitats especializados para el desarrollo de sus colonias, a cambio reciben la melaza excretada por pulgones (Way, 1963; Buckley, 1987; Sudd, 1987; Godske, 1992). En cualquier caso, los pulgones proveen de alimento a las hormigas, ya sea como presas o bien en forma de melado que ellas recolectan y frecuentemente almacenan en sus hormigueros (Covelo de Zolessi et al., 1976; Sakata, 1994; Conway, 1994). Esta afirmación, es similar con la encontrada en la investigación ejecutada, debido al porcentaje significativo de presencia de hormigas y plantas infestadas con pulgones en el valle del Mantaro.

Con relación a la distribución poblacional del número de insectos evaluado en la "retama" en las provincias muestreadas (Tabla 4), y no tiene una distribución normal como afirma Margalef, (1986); significando que hay representatividad de la población de insectos. Sin embargo Krebs (1978 y 1989), afirma que las poblaciones de insectos provenientes de un conteo, la población de insectos evaluado sigue una distribución normal, con ajuste logarítmico la curva poblacional ajustado.

Se observó que la provincia con mayor abundancia y número de individuos es Jauja con 48233 individuos, luego Concepción con 41523 individuos, seguido por Huancayo 365 II individuos y Chupaca con 28844 individuos respectivamente; siendo las familias Aphidiidae, Thripidae, Margaroridae, Formicidae, son las más abundantes; con abundancia media fueron, Braconidae, Pipunculidae, Syrphidae, Scatophagidae, Ichneumonidae, y las familias Pieridae, Coenagrionidae, Aeshnidae, Blattidae, son las que fueron menos abundantes. Y este resultado realizado la comparación de media poblacional, no existe diferencia estadística significativa. Esta característica, se afirma debido a que en las cuatro provincias de valle, las plantas de S. junceum L. comparte casi el mismo 
hábitat para el valle del Mantaro; además que la variación del clima y la altitud no son muy diferenciadas, y tienen las mismas característica ecológica. (Tabla 4).

Estimación de la diversidad de insectos en $\mathbf{S}$. junceum L. en el valle del Mantaro. Por otro lado, Gastón \& Lawton (1990) han señalado que en insectos lo más frecuente es una estrecha relación entre abundancia local y rango geográfico. Es decir, una especie localmente abundante, generalmente tiene un amplio rango geográfico. Esto no ha ocurrido con la población de insectos que hospedo S. junceum L., cuya característica fue que en un ámbito geográfico como el valle del Mantaro, que representativamente es pequeña, no existió una relación entre la abundancia local y el rango geográfico, la distribución fue similar en el ámbito evaluado, siendo muy diferente a lo planteado por Gastón \& Lawton (1990)

Se evaluaron a los insectos en S. junceum L., estimando la diversidad alfa, según la metodología desarrollada por Moreno (200I), evaluando algunos indicadores de la riqueza especifica como el índice de Margalef, los índices de abundancia proporcional con los respectivos Índices de Simpson (dominancia) y de Shannon - Wiener (equidad).

En la Tabla 6, la abundancia estimada de insectos, se obtuvo que las provincias del valle del Mantaro presentaron, una riqueza media de 56.90 familias y 3 I 48.15 individuos. Este resultado señala que la riqueza es abundante y diverso, por la relación muy favorable con la diversidad de especies vegetales del entorno S. junceum $L$. en donde crece, y mantiene una relación con la población total del número individuos para las provincias evaluadas.

Sin embargo, Sánchez (2003); afirma que una población de insectos es más abundante y diversa cuando la relación existente en el ecosistema (los factores bióticos y abióticos) son favorables y óptimo para el crecimiento de los insectos, y ello determina la abundancia de abundancia de especies. Es muy importante señalar que son las variables climáticas como son rangos óptimos temperatura, asociación vegetal existente, condiciones de humedad, y principalmente las horas sol y la presencia o no de viento, en donde inclusive las condiciones del relieve fisiográfico y de topografía son determinantes para la presencia de los insectos como indicadores de diversidad.

Se apreció en general que la abundancia del número de insectos expresaron valores en porcentaje que fue menor para Chupaca, debido a que los lugares de muestreo estuvieron localizados muy próximo a áreas en donde la actividad agrícola fue intensiva, y tuvo una respuesta sobre la abundancia de insectos posiblemente debido al efecto indirecto toxico de los residuos de los pesticidas en el agua, aire y suelo, que es utilizada de manera indiscriminada por el agricultor para el control de plagas agrícolas. Pero se observó que insectos como Aphis craccivora, Aphis cystisorum, Aphis fabae, e
Icerya. purchasi en plantas de S. junceum L. con una alta densidad poblacional, y son resistentes a los pesticidas y tolerancia a los alcaloides de quinolizidina pese a ser altamente tóxicos para insectos, como reporto Wink y Ludger ( 1990).

En cuanto a los índices de uniformidad en Chupaca, se observó mayor uniformidad, luego Huancayo y fueron menos uniformes, Concepción y Jauja probablemente por alguna influencia de las condiciones ambientales y de la interrelación de los hospedantes que crecen junto a $S$. junceum L.

En cuanto al Índice de Shannon Wiener, los insectos evaluado con el índice de Shannon-Wiener o de equidad de los insectos que hospeda $S$. junceum L., fue para la provincia de Chupaca 2,34; Huancayo 2,07; Concepción 1,62; y Jauja 1,75 respectivamente; siendo la media para el valle del Mantaro de 1,94). Este resultado indica que existe una baja diversidad y es heterogénea la población de insectos que hospedó la retama. Representa que dicha provincia no es muy diverso y heterogéneo, y representa que hay ciertos grupos de familias que no son diversos, y poco número de insectos dominantes, siendo los pulgones y los trips; y tiene una menor abundancia relativa con relación a la población total.

Con relación al índice de Simpson, los resultados de la investigación muestra valores para la provincia de Chupaca con 0.80; la provincia de Huancayo con 0.74; la provincia de Concepción con 0.58 y provincia de Jauja con 0,68. Este resultado para el índice Simpson en el valle del Mantaro de 0.70, la población de insectos en las provincias de Chupaca y Jauja tuvo una mayor dominancia y menor diversidad; mientras que la población de insectos de las provincias de Huancayo y Concepción tuvo una menor dominancia y mayor diversidad. Sin embargo existe una mayor dominancia de algunos especies fitófagas con son los pulgones y los trips. Probablemente sea, debido a que en dichas provincias se desarrolla la actividad agrícola de manera intensiva y continua, hecho que favorece la presencia de pulgones en mayor número y dominantes, con relación a las otras especies reportadas.

Con relación al índice de Margalef, los resultados señalan que representa una riqueza con un índice calculado para el valle del Mantaro 7.13; y para Chupaca 7.53; Jauja 7.09; Huancayo 7. 8 y Concepción 6.74 siendo general una alta diversidad. La provincia de Chupaca, tiene un mayor número de especies a nivel de familia, esto en razón a que la zona de muestreo en dicha provincia presentó condiciones muy favorables para la presencia de insectos en las plantas de $S$. junceum L. mientras en las provincias de Chupaca, Concepción y Huancayo, presenta características muy similares en cuanto al número de especies. Los resultados obtenidos son congruentes con la riqueza específica (S) encontrada en dichas comunidades donde la mayor riqueza se encuentra en las provincias de Chupaca y Huancayo.

Otro índice, importante determinado es Berger - Parker (d), que significa que en Concepción con un 
valor de 0,62; presentó mayores individuos por familia dominantes. La media para el valle del Mantaro fue de 0.46 se tipifica que en el resto de las provincias, las especies dominantes van disminuyendo dicha dominancia. Este resultado, confirmaron que las plantas de S. junceum L. Evaluadas estuvieron en asociación vegetal de mayor diversidad fitosociológica y diversidad de cultivos predominantes como la alcachofa y otros.

Los factores climáticos que influyen en la diversidad de insectos en $\mathbf{S}$. junceum $\mathbf{L}$. en el valle del Mantaro. La cuantificación poblacional de insectos en S. junceum L. permitió estimar la diversidad de insectos, utilizando muestras tomadas con trampas pegantes, pitfall, redada y de caída en lona; en cada provincia muestreada. Dichos métodos, por ser selectivos y no exhaustivos, existe aleatoriedad en cuanto al número de individuos y especies capturadas, lo cual influyeron en la estimación de la diversidad. En la investigación en varias muestras obtenidas para un mismo muestreo y en las mismas condiciones, se comprobó que la mayoría de las muestras siempre fueron abundantes, mientras que las especies raras, representadas con I o 2 individuos, aparecen en algunas muestras y en otras no. Esta variación aleatoria en el número de especies determina que las muestras presenten valores diferentes de diversidad de un mismo lugar como resultado de una limitación metodológica en la captura de individuos. Otro aspecto que influyo es que las poblaciones de insectos cambian con la ocurrencia del tiempo expuestos principalmente a los factores climáticos. trampas pegantes, pitfall, redada y de caída en lona.

Modelos de distribución de insectos en S. junceum L. en el valle del Mantaro. Con relación a la distribución determinado, fue el modelo de series logarítmicas de Fisher, Corbet \& Williams (1943), que representó matemáticamente la relación entre el número de especies y el número de individuos que estuvieron presentes. Las Figuras 8, 9, 10 y || muestra la distribución de abundancia que sigue el modelo de la serie logarítmica adoptado por Fisher Corbet \& Williams (1943). Sin embargo, según el modelo de Preston (1948) o modelo lognormal predijo que la cantidad de especies presentes en cada provincia muestreada tuvo una relación Gausiana o Normal con el logaritmo de la abundancia en forma de campana. Asimismo, dicho modelo estimo los índices de diversidad que mostraron muestran una distribución log normal, siendo similar al modelo aplicado por Magurran, (1988); Krebs, (1978) y Krebs, (1989).

Influencia del clima en población de insectos en S. junceum, en el valle del Mantaro. La distribución de los insectos que en la "retama" (Tabla 9), y según el clima observado (Figura |3), de enero a diciembre del 2010 (Senamhi, 2010). Se presentaron individuos en varios estados metamórficos, sin embargo solo se consideraron estado imago, para facilitar en conteo y la identificación taxonómica. La ocurrencia del clima, fue muy variable observando la temperatura más baja en junio con $11,10^{\circ} \mathrm{C}$ y la temperatura máxima fue $13,46^{\circ} \mathrm{C}$ en el mes de enero. Mientras que la precipitación fue las más baja en el mes de junio con 3, 18 mm y la alta fue en el mes de enero con 145,34 mm. La precipitación, tuvo un efecto en la presencia principalmente de los pulgones y trips. La humedad relativa influyó significativamente en la población de insectos observada, siendo para el periodo evaluado de valor mínimo 60,60\% en el mes de setiembre y 71,60\% en el mes de enero. Mientras que las horas sol ocurrida fue de valor mínima mensual de 122,6 horas sol en febrero y el valor máximo 274,85 horas sol.

Cabe señalar que las investigaciones realizadas por Chen y Tsong (2002) e Ibrahim (1985), enfatizaron el efecto de la temperatura en el desarrollo de los diferentes estadios de homópteros como la queresa, y Yu An Lin (2005) que estudió la fluctuación poblacional de A. yabunikkei Kuwana y A. murrayae Takahashi (Hemíptera: Diaspidae) en plantas de cítricos en el área de Taipei, Taiwán, demostraron que hubo una correlación entre la temperatura y la presencia de todos los estados para las queresas, resultando que un incremento de la temperatura acelera la proporción de todos los estados y contrariamente ocurre cuando la temperatura disminuye. Pero Ibrahim (1985) enfatizó que cuando la temperatura disminuye el tamaño de $\mathrm{S}$. coffeae incrementa, en los meses más fríos hubo mayor densidad poblacional de pulgones y trips, por lo que se observó una mayor tasa de reproducción de individuos en la "retama". También afirma Beingolea (1969), la queresa y pulgones son especies adaptadas a climas templados o por lo menos más tolerante al frío, como ocurrió para $S$. junceum L. que está bien distribuido en el valle del Mantaro.

Sin embargo, los factores exógenos como la temperatura, la humedad relativa, la precipitación y la luz del sol total por lo general influyen en la población de insecto enormemente, como afirma (Kisimoto y Dyck, 1976), cuyo resultado fue similar a la presencia de insectos en S. junceum L. en el valle del Mantaro.

Durante la evaluación se observó que la población de pulgones varió en la "retama", debido a la variación de los parámetros de tiempo, como algunos rastros de lluvia, rocío y horas sol dieron lugar a la alta nubosidad que favoreció la incidencia de pulgones considerablemente. Esto concuerda según la investigación realizado por Kumar et al., (1997) que informaron que la temperatura media de $18,060{ }^{\circ} \mathrm{C}$ (22,810 máximo y mínimo $13,31^{\circ} \mathrm{C}$ ) bajo la influencia de la alta humedad relativa con el rango de $80,71 \%$ a $86,5 \%$ siempre favorecen la incidencia de pulgones, comportamiento climático muy similar a la observada principalmente para la temperatura en el valle del Mantaro.

En la Figura 14, se observa la ocurrencia poblacional de los insectos en S. junceum L. durante el periodo evaluado, y se ubicaron con mayor frecuencia los pulgones y los trips. Algunos individuos mostraron cierto 
comportamiento preferencial a la sombra evadiendo el efectos de la luz diurna, así como localizándose en partes florales suculentas y brotes tiernos, para facilitar su alimentación.

La fluctuación poblacional de los insectos observados muestran la relación que tuvieron con los factores ambientales; los datos indicaron que la humedad relativa (\%) y las horas sol (hs) tuvieron una correlación negativa con la población de insectos en S. junceum L., reportando una mayor presencia de fitófagos en periodos secos. La precipitación influyo significativamente en los pulgones y "trips", por el efecto físico de lavado y ahogamiento reduciendo el número de individuos; mientras la temperatura, su influencia no fue determinante en periodos más fríos en razón a que los insectos son de comportamiento poiquilotermos y ectotérmicos, como refiere Sánchez (2003), y Beingolea (1969); siendo característica muy similar a la observada por Yu An (2005). La variable horas sol, tuvo una correlación positiva, aumentando el número de individuos cuando las horas sol incrementaron como ocurrió de marzo a agosto del 2010, y contrariamente la población disminuía cuando las horas sol como ocurrió en los meses de mayor pluviosidad.

En la Figura 15, se observa la distribución normal de la regresión múltiple para la población normal de insectos presente en la $\mathrm{S}$. junceum L. siendo la ecuación $N^{0}$ Ins. $=120357.827+123.067 * P P-1513.823 * H R$ - 81.736*HS. Determinando que el incremento de la precipitación influyó en la presencia de mayor población de insectos en la "retama", y mientras que la humedad relativa y horas sol, tuvieron un efecto en la disminución de la población.

Según investigación realizada por Nasir y Ahmad (200l), así como Aheer et al., (2008) informó que la temperatura tenía un papel importante y positivo en densidad de la fluctuación de los pulgones. Por otra parte, humedad relativa reveló una correlación negativa y significativa con la población de pulgones. También informaron que la densidad de los pulgones tuvo correlación negativa no significativa con la precipitación. Pero Wains, (2008) reportaron una correlación negativa significativa entre la densidad de pulgón y lluvias durante el año 2005. Los resultados logrados, difieren con lo reportado para el valle del Mantaro, durante el periodo evaluado. En la investigación ejecutada, existe correlación significativa estadística de los factores climáticos de la precipitación $(p=0,782)$; humedad relativa $(p=0,55 \mathrm{I})$ y horas sol $(p=-0,8 \mid 4)$ existe con el número de insectos observado en la "retama" (Tabla 10). También se explica que los factores climáticos influyen en la variación poblacional de insectos en la "retama" ( $p=0,000)$, del análisis de varianza de la regresión lineal múltiple estimada (Tabla II). Sin embargo la variación poblacional de insectos está relacionada a la diversidad con la abundancia y riqueza específica, por lo que se acepta la hipótesis alterna de la investigación.
Cambios en la composición, estructura y diversidad de insectos en $S$. junceum $L$. por influencia del clima en el valle del Mantaro LoS insectos representan casi la mitad de la biodiversidad descrita hasta ahora (Speight et al. 1999) y son piezas centrales en la estructura y función de los ecosistemas (Crawley, 1983). Debido a su estrecha relación con plantas hospederas, insectos herbívoros sufren los efectos directos e indirectos del cambio climático a través de los cambios experimentados en las plantas hospederas. El cambio climático global afecta los patrones de riqueza, diversidad y abundancia (Thuiller et al., 2005, Kazakis et al., 2007). Los insectos desempeñan un papel importante en los servicios de los ecosistemas, en calidad de herbívoros, polinizadores, depredadores y parasitoides, y los cambios en su abundancia y diversidad tienen el potencial de alterar los servicios que prestan (Hillstrom y Lindroth 2008).

Los estudios han demostrado que el aumento de temperatura tiende a tener efectos positivos sobre los insectos (Bale et al., 2002), insectos especialmente multivoltinas en ecosistemas templados, el cambio climático puede actuar directamente sobre un insecto ya sea como un factor de mortalidad o mediante la determinación de la tasa de crecimiento de insectos y/o desarrollo. La temperatura puede alterar la duración del ciclo de vida del insecto, voltinismo, la densidad de población, el tamaño, la composición genética, el grado de aprovechamiento de las plantas huésped (en tiempo y espacio) y la distribución geográfica (Bale et al., 2002).

En la investigación realizada, la variación de abundancia y diversidad de los insectos evaluado en $S$. junceum $L$. en el valle del Mantaro, se debió a algunas condiciones extremas que ocurrió en condiciones de clima seco, por la disminución de la precipitación en algunos meses, una alta incidencia solar y baja humedad relativa durante la mayor parte del año, como sostiene Reis (1976); coincidiendo ampliamente los resultado observado para el valle del Mantaro.

Sin embargo Ambrosie, (2004), afirma que la humedad del medio ambiente regula directamente la población del insecto y sus efectos son fuertemente modificados por la temperatura. Los Insectos sobreviven en rango óptimo de humedad y temperatura. La mortalidad puede ocurrir solamente en condiciones extremas de humedad que son inadecuados para las fases activas de muchas especies de insectos.

Los resultados de la investigación demuestran, que factores climáticos como la humedad relativa y horas sol, que tiene una relación con la temperatura influyeron significativamente en la abundancia y diversidad de especies de insectos que hospedo S. junceum L. en el valle del Mantaro; siendo factores que determinan la tasa de reproducción y desarrollo de los insectos, como sostiene Fazal et al., (20/2), en una investigación realizada en Pakistán, y está estrechamente relacionada con la abundancia y heterogeneidad de los insectos evaluado.

En la investigación realizada, se planteó la hipó- 
tesis, que los factores climáticos influyen en la diversidad de insectos en la "retama". En la Figura 17 y Tabla 13, la regresión estimado tiene nivel de significación estadística $(p=0,00 \mathrm{I})$ donde la humedad relativa influyó sobre el índice de Margalef estimado, y la correlación es negativa.

Identificación de insectos en S. junceum L. Algunas especies identificadas constituyen plagas claves de S. junceum L. como Aphis cystisorum, Aphis craccivora y Aphis fabae, siendo similar la reportada por Vilca y Vergara (20I I). También hubo la presencia de otras especies de insectos que son plagas claves y potenciales de cultivos agrícolas.

La variación de los factores climáticos durante el año 2010, con el incremento de la precipitación en los meses de enero a marzo, y luego manifestar un clima seco y de menor precipitación, mayor frio e irradiación solar en los meses de marzo a agosto. Esta variación a un clima seco y frio durante la mayor parte de año y de menor actividad agrícola, indujo a otros insectos que son hospedantes en cultivos agrícolas, ornamentales y forestales migraran a la "retama", que es una planta semileñoso, siempre verde durante la mayor parte del año, y esto favoreció las presencia de muchos insectos que fueron identificados.

Catalán et al., (2006), reporto para la "retama" en Cusco A. craccivora (Koch); registrando predadores como Shyrpus sp., Platycheirus sp., y Toxomerus sp. (Syphydae: Díptera; Eriopis peruviana; $H$. convergens (Coccinellidae: Coleoptera.; Parasitoide Praeon sp. (Braconidae: Himenóptera); siendo similares las especies identificadas para el valle del Mantaro.

\section{Conclusiones}

La población de insectos en S. junceum L. en el valle del Mantaro, cuya composición y estructura muestra una baja diversidad y heterogénea, con pocas especies dominantes y con mayor abundancia poblacional constituido por los pulgones y "trips" principalmente. La población de insectos en $S$. junceum $L$, fue mayor, cuando la precipitación tuvo un incremento, y cuando la humedad relativa y las horas sol disminuyeron en el valle del Mantaro; siendo los factores climáticos que influyeron significativamente.

La humedad relativa influyo en diversidad estimada del índice de Margalef; hubo menor diversidad, mayor dominancia y heterogeneidad de la población de insectos en S. junceum L. cuando hubo un incremento de la humedad relativa.

Se confirmó la identificación de 10 órdenes, 32 familias, 35 géneros y 16 especies por el Servicio Nacional de Sanidad Agraria

\section{Referencias}

Aguilar, C. (2009). Diversidad de la entomofauna en Spartium junceum L. en el valle del Mantaro. Tesis
Ingeniero Forestal y Ambiental. Facultad de Ciencias Forestales y del Ambiente. Universidad Nacional del Centro del Perú. Huancayo. Perú. 59 p.

Aheer G.M., Ali A., Munir M. (2008). Abiotic factors effect on population fluctuation of alate aphids in wheat. J. Agric. Res. 46 (4): 367-37I.

Alvarado, A. y Huamán, D. (2008). Control integrado de Aphis fabae en Senna hookeriana y Aphis craccivora Koch en Spartium junceum L. Tesis Ingeniero Forestal y Ambiental. Facultad de Ciencias Forestales y del Ambiente. Universidad Nacional del Centro del Perú. Huancayo. Perú. 109 p.

Ambrosie, D.P. (2004). The Insects: Structure, Function and Biodiversity. pp. 362-59. 3rd edi. Kalyani publishers, New Delhi, India..

Arroyo, J. (20 I I). Tendencias y cambio del clima del valle del Mantaro mediante los análisis de índices de Precipitación Efectiva y Temperatura Eficiente. Apunt. Cienc. Soc. 201 I; OI (0I).

Bale, J. S., G. J. Masters, I. D. Hodkinson, C. Awmack, T. M. Bezemer, V. K. Brown, J. Butterfield, A. Buse, J. C. Coulson, J. Farrar, J. E. G. Good, R. Harrington, S. Hartley, T. H. Jones, R. L. Lindroth, M. C. Press, I. Symrnioudis, A. D. Watt, and J. B. Whittaker. 2002. Herbivory in global climate change research: direct effects of rising temperature on insect herbivores. Global Change Biology 8: $1-16$.

Baltazar, H. (2007). Estudio del afido negro de Spartium junceum - Tres de Diciembre - Chupaca. Informe Final. Instituto de Investigación. Facultad de Ciencias Forestales y del Ambiente. UNCP. Huancayo. 21 p.

Beingolea, O. (1969). Notas sobre la biología de Saissetia oleae Bern. (Hom.: Coccidae), "Queresa negra del olivo", en laboratorio y en el campo. Rev. Per. de Ent., I2(I): 130-136.

Borror D., de Long D. \& Thriplehorn C. (1995). An introduction to the study of insects. Saunders College Publishing. 6th Ed. USA. 827 p.

Brack, A. y Mendiola, C. "Ecología del Perú". (2004). Editorial Bruño, Lima. Pág. 7-81, 83-445, 447-493 Briceño V., Hernández F., Moreno Z. 2005. Diversidad en conucos agroforestales en la comunidad indígena de Coromoto de Cuao, Estado de Amazonas, Venezuela XIX Congreso Venezolano de Entomología. Entomotropica 2005: Vol. 20(2): I27-204. Venezuela. ISSN $1317-5262$.

Buckley, R. C., (1987). Ant - Plant-Homopteran interactions. Adv. Ecol. Res., 16: 53-85. Cannon, R. J. C. 
1998. The implications of predicted climate change for insect pests in the UK, with emphasis on non-indigenous species. Global Change Biology 4:785-796.

Catalán, J., Camarero, L., Felipe, M., Pla, S., Ventura, M., Buchaca, T. 2006. High mountain lakes: extreme habitats and witnesses of environmental changes. Limnetica 25: 55।-584.

Chen, Y.L. \& Tsong, H. S. (2002). Effects of temperature on development of the hemispherical scale, Saissetia coffeae (Walker) (Homoptera: Coccidae), and its occurrence on cycad (Cycas taiwanian Carr.). Formosan Entomologist 22(I): 65-74.

Clench H. (1979). How to make regional list of butterflies: Some thoughts. J. Lepid. Soc. 33, 216-31. Coley, P. D. 1998. Possible effects of climate change on plant/herbivore interactions in moist tropical forests. Climatic Change 39:455-472.

Conway, J. R., (1994). Honey Ants. American Entomologist, 40 (4): 229-234. Crawley M. J. 1983. Herbivory. The dynamics of animal-plant interactions. Oxford, Blackwell Scientific Publications, 437 p.

Covelo de Zolessi, L.; Petrone de Abenante, y. Gonzales, L. A., (1976). Descripción y observaciones bioetológicas sobre una nueva especie de Brachymyrmex (Hymenoptera:Formicidae). Revista de Biología del Uruguay, 4 ( I): 21 - 43.

Delfino, M. A. y Buffa, L. M. (2000). Algunas interacciones planta-áfido-hormiga en Córdoba (Argentina). Zool. baetica, II: 3-15, 2000. Fazal, S.; Manzoor, and Abdul-Sattar. 2012. A. impact of abiotic factors on insect diversity of at Lawrence Garden, Lahore. Pakistan Journal of Science. Vol. 64 No. 2 June.

Fisher, R. A.; Corbet, A. S. y Williams, C. B. (1943). The Relation Between the Number of Species and the Number of Individuals in a Random Sample of an Animal Population. J. Anim. Ecol., 12, 42-58.

Frost, C. J., and M. D. Hunter. (2004). Insect canopy herbivory and frass deposition affect soil nutrient dynamics and export in oak mesocosms, Ecology, 85, $3335-3347$

Ganho N.G. \& Marinoni R.C. (2003). Fauna de Coleoptera no Parque Estadual de Vila Velha, Ponta Grossa, Paraná, Brasil. Abundancia e riqueza das familias capturadas através de armadilhas malaise. Revista brasileira de Zoologia. 20: 727-736.

Gaston, K. J. \& Lawton, J. H., (1990). Effects of scale and habitat on the relationship between regional distribution and local abundance. Oikos, 58: 329-335.
Gaviño de la Torre, G.; Suarez L., C.; Figueroa T., H. (2004). Técnicas biológicas selectas de laboratorio. Segunda Edición. Limusa Noriega Editores. Mexico. $308 \mathrm{p}$.

Godske, L. (1992). Aphids in nests of Lasius flavus F. in Denmark. II: Population dynamics (Aphodoidea, Anoeciidae \& Pemphigidae; Hymenoptera, Formicidae) Ent. Meddr., 60 (I): $21-26$

González-Oreja, J. A.; De la Fuente-Díaz - Ordaz, A. A.; Hernández-Santín, L.;, Buzo-Franco, D. \& Bonache - Regidor, C., (20l0). Evaluación de estimadores no paramétricos de la riqueza de especies. Un ejemplo con aves en áreas verdes de la ciudad de Puebla, México. Animal Biodiversity and Conservation, 33.1: $31-45$.

Henriksson, J. et al., (2003). Effects of host shading on consumption and growth of the geometrid Epirrita autumnata: interactive roles of water, primary and secondary compounds. Oikos 103: 316

Hernández R., Fernández C. y Baptista P. (2006). Metodología de la Investigación. (4a. Ed.). México: McGraw-Hill/ Interamericana Editores.

Hillstrom M.L. and Lindroth R. L. (2008). Elevated atmospheric carbon dioxide and ozone alter forest insect abundance and community composition. Insect Conserv Div I : 233-24l.

Holdridge, L. (1987). Ecología basada en zonas de vida. Instituto Interamericano de Cooperación para la Agricultura, San José, Costa Rica. 216 p.

Hutcheson K., (1970). A test for comparing diversities based on the Shannon formula. J. theor. Biol., US. I5I- 154 p.

lannacone J., Alayo M. \& Sánchez J. (2000). Biodiversidad de la artropofauna del Bosque Zárate, Lima-Perú, empleando tres técnicas de censo. Wiñay Yachay (Perú). 4: 27-46.

Ibrahim, A. G. (1985). The effects of temperature on the development of hemispherical scale, Saissetia coffeae (Walker). Pertanika 8: 381-386.

Kazakis G, Ghosn D, Vogiatzakis N, Papanastasis VP. (2007). Vascular plant diversity and climate change in the alpine zone of the Lefka Ori, Crete. Biodiv Conserv 16: 1603-1615.

Kisimoto, R. and Dyck, V.A. (1976). Climate and rice insects. In proceedings of symposium on climate and Rice, International Rice Research Institute, Manila. pp. 36|-39|. 
Klingauf, F. A. (1987). Host plant finding and acceptance, p. 209 - 220. In A. K. Minks and P. Harrewijn (eds.), World crop pests, aphids: their biology, natural enemies and control, vol. 2A. Elsevier, New York.

Krebs, C.J. (1978). Ecology: The experimental analysis of distribution and abundance (Second edition ed.). Harper \& Row, New York. 678 p.

Krebs, C. J. (1989). Ecological methodology. Menlo Park, Benjamin/ Cummings. $58 \mathrm{lp}$.

Kuehl, R. 200I. Diseño de Experimentos. Thomson Editores, S.A. de C.V. México D.F. 666 p.

Kumar, A., Tripathy, M.K. and Srivastava, K.M. ( 1997). Population dynamics of black bean aphid (Aphis craccivora Koch.) in different genotypes of Indian bean (Dolichos lablab) in relation to weather parameters at Kanpur. UP. Environ. Ecol. I5(2): 318-321.

Lightfoot, D. (2006). Climate Change and Rangeland Insects. Department of Biology The University of New Mexico Albuquerque, NM 87 I I BLM Global Climate Change Research Program, LTER, NPS, USGS.

Halffter, G. (1992). “QQué es la biodiversidad?” In: La diversidad biológica de Iberoamérica. Acta Zoológica Volumen Especial. CYTED-D, Instituto de Ecología, Secretaría de Desarrollo Social, México. 3 - 24 pp. Referenciada. 09/07/2009. Disponible: http://www. gruponahise.com/simposio/papers\%20pdf/25\%20 OSCAR\%20DEL\%2OPINO.P

Magurran, E. A. (1988). Ecological Diversity and Its Measurement. Princeton University Press. 192 p.

Margalef. R. (1995). Ecología. Ediciones Omega, SA. Barcelona. España. 320 p.

Ministerio de Agricultura. (2007). Encuesta por muestreo (EPM). Resultados región Sierra 2006. Departamento: Ancash. Dirección General de Información Agraria. Ministerio de Agricultura. 2007. Producción de horticultura Por Perú. 622 pg.

Moreno, C. E. (200I). "Métodos para medir la biodiversidad”. M \& T-Manuales y tesis SEA, Vol. I. Zaragoza, 84 pp. Referenciada: 15/04/2009. Disponible: http:// www.google.com.bo/search?hl=es\&q=metodos+paratmedir+biodiversidad.

Nasir S., Ahmad F. (200I). Correlation between wheat aphid population and abiotic factors. Pak. Entomol. 23 ( I): 23-25.

Oyvind Hammer. (20/2). Past. Paleontological Statistics. Version 2.17. Reference manual. Natural History Museum. University of Oslo. ohammer@nhm.uio.no.
1999-2012. Disponible en http://nhm2.uio.no/norlex/past/docl.html Preston, F. W., 1948. The commonness, and rarity, of species. Ecology, 29: 254-283.

Quisenbery S.S. \& Xinzhi N. 2007. Feeding injury. Pp. 331- 352. In: Van Emdem, H. F. and Harrington, R., Eds.2007. Aphids as Crop Pests. CAB International. Wallingford.

Raspudić, E. et al., (1990). Distribution of Thysanoptera species and their host plants in Croatia. Acta agriculturae Slovenica, 93 - 3. 275 - 283.

Reis, A.C. 1976. Clima da Caatinga. Anais da Academia Brasileira de Ciências 48: 325-335.

Sánchez, G. (2003). Ecologia de Insectos. Universidad Nacional Agraria La Molina. Departamento de Entomología y Fitopatología. Lima. Perú. 283 p.

Sánchez, G. (2005). Comunicacional personal. Escuela de Posgrado. Universidad Nacional Del Centro Del Peru.

Santisteban, J. y Aldana G., (2005). "Manual de Prácticas de Entomología General", Departamento de Entomología y Fitopatología, Escuela de Post - Grado de la Universidad Agraria La Molina.

Sakata, H. (1994). How an Ant Decides to Prey on or to Attend Aphids. Res. Popul. Ecol. 36 ( I): 45-5I.

Servicio Nacional de Meteorología e Hidrología (Senamhi). (2008). Hoja informativa datos clima valle del Mantaro 1998-2007. I p.

Servicio Nacional de Sanidad Agraria (Senasa). 2006. Informe técnico de la plaga de la retama en el valle del Mantaro. 22 p.

Servicio Nacional de Sanidad Agraria (Senasa). (20/0). Reporte dato clima valle del Mantaro. Informe preparado para Hernán Baltazar.

Servicio Nacional de Sanidad Agraria (Senasa). (2008). Hongos e Insectos forestales en el Perú. Ministerio de Agricultura. Dirección de Sanidad Vegetal. Sub Dirección de Análisis de Riesgo y Vigilancia Fitosanitaria. $42 \mathrm{p}$.

Soberón, J. \& Llorente, J. (1993). The use of species accumulation functions for the prediction of species richness. Conservation Biology, 7: 480-488.

Speight MR, Hunter MD, Watt AD.(1999). Ecology of Insects - concepts and applications. Oxford, BlackweII Science, 340 p.

Stefanescu, C., Peñuelas, J. \& Filella, I. (2003). Effects of 
climate change on the phenology of butterflies in the northwest Mediterranean Basin. Global Change Biology, 9, | 494- 1506.

Sudd, J. H. (1987). Ant Aphid Mutualism. En: Minks \& Harrewijn (Eds), Aphids, their Biology, Natural Enemies and Control, Vol. 2A, pp. 355-365. Amsterdam. Elsevier.

Tazza, C y Suárez, L. (2004). Cambio climático en el valle del Mantaro - Huancayo - Perú. Cambios Climáticos, Recursos Hídricos, Geo-Riesgos y Desastres Naturales 3-50. Instituto de Investigaciones para el Desarrollo Tecnológico. Huancayo - Perú.

Thomanzini, M. J.; Thomanzini, A. P. B. W. (2002). Levantamento de insetos e análise entomofaunística em floresta, capoeira e pastagem no Sudeste Acreano. Rio Branco: Embrapa Acre, 4 I p. (Boletim de Pesquisa e Desenvolvimento, 35).

Thuiller W, Lavorel S, Araujo MB, Sykes MT, Prentice C. (2005). Climate change threats to plant diversity in Europe. PNAS 102: 8245-8250.

Vilca, K. y Vergara C. (20II). Los afidos (Hemíptera: Aphididae) en el Callejón de Huaylas - Ancash, Perú. Ecología Aplicada, 10(2).

Wains M.S., Aziz-ur-Rehman, Latif M., Hussain M. (2008) Aphid dynamics in wheat as affected by weather and crop planting time. J. Agric. Res. 46 (4): 36I-366.

Way, M. J., (1963). Mutualism between ants and honeydew-producing Homóptera. Ann. Rev. Entom., 8: 307-344

Wilf, P. and Labandeira, C. C. (1999). Response of plant-insect associations to Paleocene-Eocene warming. Science 284:2153-2156.

Wink, M. and Ludger, W. (1990). Storage of Quinolizidine Alkaloids in Macroshiphun albifrons and Aphis genistae (Homoptera:Aphididae). Entomol. Gener. |5(4):237-254. Stuttgart |99|-0I.ISSN:017|-8|77. Institut fur Pharmazeutizche Biologie, Universitat Heidelberg, Germany.

Yu An, L. (2005). Population Ecology of Aulacaspis yabunikkei Kuwana and Aulacaspis murrayae Takahahsi (Hemiptera: Diaspididae) in Taipei Area. [On Line]. Electronic Theses \& Dissertations Services. Graduate Institute of Entomology. National Taiwanian University. Disponibility in: [www.etds.lib.ntu.ed.tw]. Visited: [20-09-10]. 